\title{
Symmetries of spacetimes embedded with an Electromagnetic String Fluid
}

\author{
Michael Tsamparlis* \\ Faculty of Physics, Department of Astronomy-Astrophysics-Mechanics, \\ University of Athens, Panepistemiopolis, Athens 157 83, Greece. \\ Antonios Mitsopoulos ${ }^{\dagger}$ \\ Faculty of Physics, Department of Astronomy-Astrophysics-Mechanics, \\ University of Athens, Panepistemiopolis, Athens 157 83, Greece. \\ Andronikos Paliathanasis ${ }^{\ddagger}$ \\ Instituto de Ciencias Físicas y Matemáticas, \\ Universidad Austral de Chile, Valdivia, Chile. \\ Institute of Systems Science, Durban University of Technology \\ Durban 4000, Republic of South Africa.
}

\begin{abstract}
The electromagnetic string fluid (EMSF) is an anisotropic charged string fluid interacting with a strong magnetic field. In this fluid we consider the double congruence defined by the 4-velocity of the fluid $u^{a}$ and the unit vector $n^{a}$ along the magnetic field. Using the standard $1+3$ decomposition defined by the vector $u^{a}$ and the $1+1+2$ decomposition defined by the double congruence $u^{a}, n^{a}$ we determine the kinematic and the dynamic quantities of an EM string fluid in both decompositions. In order to solve the resulting field equations we consider simplifying assumptions in the form of collineations. We decompose the generic quantity $L_{X} g_{a b}$ in a trace $\psi$ and and a traceless part $H_{a b}$. Because all collineations are expressible in terms of the quantity $L_{X} g_{a b}$ it is possible to compute the Lie derivative of all tensors defined by the metric i.e. the Ricci tensor, the Weyl tensor etc. This makes possible the effects of any assumed collineation on the gravitational field equations. This is done as follows. Using relevant identities of Differential Geometry we express the quantity $L_{X} R_{a b}$ where $R_{a b}$ is the Ricci tensor in terms of the two irreducible parts $\psi, H_{a b}$. Subsequently using the gravitational field equations we compute the same quantity $L_{X} R_{a b}$ in terms of the Lie derivative of the dynamic variables. We equate the two results and find the field equations in the form $L_{X}$ Dynamic variable $=F\left(\psi, H_{a b}\right.$, dynamic variables $)$. This result is general and holds for all gravitational systems and in particular for the EMSF. Subsequently we specialize our study at two levels. We consider the case of a Conformal Killing Vector (CKV) parallel to $u^{a}$ and a CKV parallel to $n^{a}$. Finally we solve the resulting field equations in the first case to the Friedman Robertson Walker (FRW) spacetime and in the second case for the Bianchi I spacetime. In the latter case we find a new solution of the gravitational field equations.
\end{abstract}

\section{Introduction}

Relativistic magnetohydrodynamics is the main theory which can describes various phenomena in modern astrophysics, for some recent reviews we refer the reader in [1, 2, 3, 4, When a charged plasma enters a strong magnetic field it is possible the pressures along the magnetic field and perpendicular to the magnetic field to be unequal. This results in a physical system which we call an anisotropic electromagnetic string fluid (EMSF).

\footnotetext{
*Email: mtsampa@phys.uoa.gr

$\dagger$ Email: antmits@phys.uoa.gr

†Email: anpaliat@phys.uoa.gr
} 
In the present work we study the dynamics of an isolated anisotropic gravitating fluid which for the observers $u^{a}\left(u_{a} u^{a}=-1\right)$ has the energy momentum tensor of the form

$$
T_{a b}=\mu u_{a} u_{b}+p_{\|} n_{a} n_{b}+p_{\perp} p_{a b}
$$

where

- $n^{a}$ is a unit spacelike vector which is characteristic of the fluid

- $\mu$ is the matter density of the fluid observed by the observers $u^{a}$

- $p_{a b}$ is the tensor projecting normal to both the vectors $u^{a}$ and $n^{a}$ defined by the relation:

$$
p_{a b}=h_{a b}-n_{a} n_{b}
$$

where

$$
h_{a b}=g_{a b}+u_{a} u_{b}
$$

is the tensor projecting normal to the vector $u^{a}$.

This type of fluid is a special case of a string fluid. A general string fluid is defined [5, 6] as a fluid consisting of the mixture of a general fluid with energy momentum tensor $T_{a b}$ and a second fluid which is characterized by an antisymmetric tensor field $F_{a b}$ with energy momentum tensor ${ }_{F} T_{a b}$ of the form

$$
{ }_{F} T_{a b}=\sigma n_{a} n_{b}
$$

where $\sigma$ is a function and the spacelike vector $n^{a}=F^{a b} u_{b}$. For certain types of $T_{a b}$ it is possible that the energy momentum tensor ${ }_{F} T_{a b}+T_{a b}$ of the string fluid has the form (1).

There have been many types of sting fluids considered in the literature involving different combinations of the two fluids (see $91,[10,[11$, [12, [13, ,14]). The recent works on the topic study the string fluid mainly from the point of view of thermodynamics by considering conserved currents and the corresponding chemical potentials including - in most of the studies - the entropy and the temperature. They seem go make no extensive use of the gravitational field equations and concentrate rather on the catastatic equations in order to complete the set of the field equations. The problem with this approach is that the geometry of spacetime does not enter explicitly into the study therefore one cannot use the results for the various important spacetime considered in General Relativity and particularly on the various cosmological models.

Our approach follows the line of older research on string fluids where emphasis was given in the macroscopic study of a gravitating string fluid using geometric methods and especially collineations to simplify the dynamical equations. By a collineation or geometric symmetry of a geometric object $A$ defined by the metric (not necessarily a tensor) we mean an equation of the form $L_{X} A=B$ where $B$ is a tensor field with the same number and the same symmetries of indices as $A . X$ is the vector generating the collineation. It can be shown (see [33]) that the quantity $L_{X} A$ can be expressed in terms of the fundamental quantity $L_{X} g_{a b}$. Therefore it is possible to characterize all collineations in terms of the quantity $L_{X} g_{a b}$. Furthermore we may consider the decomposition of the quantity $L_{X} g_{a b}$ in a trace and a traceless part as follows

$$
L_{X} g_{a b}=2 \psi g_{a b}+H_{a b}
$$

where $H_{a b}$ is traceless and characterizes each collineation in terms of the tensors $\psi, H_{a b}$.

Of particular interest is the quantity $L_{X} R_{a b}$ which enters the gravitational field equations because allows one to express these equations in terms of the quantity $L_{X} g_{a b}$ (or the tensors $\psi, H_{a b}$ ) so that for each particular collineation one simply replaces the appropriate expression of $L_{X} g_{a b}$ and obtains the gravitational equations in a form that already incorporates the collineation.

Concerning the study of a gravitating string fluid using collineations this must be based on a scenario which shall be independent of the particular type of the collineation. In addition it should be systematic in the sense that it will describe the steps one has to follow in order to get to the required answers in the easiest and safe way. A first attempt to develop such a scenario was done in [29], however we feel that it must be stated again briefly in a more systematic way. The proposed scenario we apply in this work is general and has as follows.

The study of a string fluid requires two main vector fields, the four-velocity $u^{a}$ and the vector filed $n^{a}$ $\left(u^{a} n_{a}=0\right)$ describing a dynamic variable of the fluid. These vector fields define a set variables which are classified in two sets

a. Kinematic variables which are due to the vector field $u^{a}$ 
b. Physical variables which are defined by the vector field $n^{a}$.

The kinematic and the dynamical variables are not independent because they are constrained by the following conditions

i. Certain geometric identities which the vector fields $u^{a}, n^{a}$ must satisfy (e.g. Ricci identity etc) 0

ii. The gravitational field equations $G_{a b}=T_{a b}+{ }_{F} T_{a b}$ from which results the conservation law $\left(T^{a b}+{ }_{F} T^{a b}\right)_{; b}=$

ii. The field equations of the tensor field $F_{a b}$ together with the constraint $F_{; b}^{a b}=0$.

The standard way to define the dynamic variables of all fluids is the covariant decomposition of the tensor fields defining the fluid by means of the $1+3$ decomposition defined by the projection tensor $h_{a b}$ in (3) and the $1+1+2$ decomposition defined by the screen projection tensor $p_{a b}$ in (2).

Subsequently all above conditions a.-b. and i. - iii. must be expressed $1+3$ and $1+1+2$ decomposed in order to define the physical variables and subsequently to obtain a set of equations which incorporate fully the dynamics of the string fluid in all spacetimes and for all possible collineations. As expected the set of the final equations is not enough for the determination of all dynamical variables and one has to introduce new assumptions which are the equations of state and possibly other additional physical assumptions.

We demonstrate the above scenario to a particular string fluid which has been considered in various forms in the literature, see [5, 6] and references therein. This string fluid consists of a mixture of a charged perfect fluid interacting with an electromagnetic field which is described in the RMHD approximation with vanishing electric field and infinite conductivity. This particular string fluid in the following we shall call the electromagnetic string fluid (EMSF). Physically an EMSF occurs for example when a charged plasma enters a strong magnetic field where it is possible the pressures along the magnetic field and perpendicular to the magnetic field are unequal. In this case the EM field contains a magnetic field only $H^{a}$ and $F_{a b}=\eta_{a b c d} H^{c} u^{d}$ whereas the vector $n^{a}=H^{a} / H$.

Our purpose is to provide a useful tool for the people who would like to work on this direction in the future. Inevitably in this approach we shall have to recall pieces of results from previous works which have required long and tedious calculations which there is no point to be repeated (see [27]).

The structure of the paper is as follows. In Section 2 for the convenience of the reader we review briefly the definition of physical variables of the string fluid using the the $1+3$ and $1+1+2$ decompositions. The EMSF is defined in Section 3 where we discuss the $1+1+2$ decomposition of Maxwell equations. Furthermore, the gravitational field equations for the model are presented in Section 4 In Sections 5 and 6 we find the kinematic and the dynamic conditions for the gravitational field equations when the spacetime admits a timelike or a spacelike CKV. We demonstrate our results in Section 6.3 where we apply the constraint conditions in the case of Bianchi I spacetime. Finally, in Section 7 we draw our conclusions.

\section{The definition of the physical variables}

The definition of the dynamical variables of a relativistic fluid is done with the use of the $1+3$ and the $1+1+2$ decomposition of the characteristic fields $u^{a}, n^{a}$ of the fluid. The $1+3$ decomposition generated by $u^{a}$ can be found among others in the early (and excellent) paper of Ellis [32] whereas the $1+1+2$ in [23, [27] kai alla. In the following we review briefly the application of these decompositions for the case of a string fluid.

\subsection{The $1+3$ decomposition}

Consider a spacetime with metric $g_{a b}$ and a fluid of observers with four-velocity $u^{a}\left(u^{a} u_{a}=-1\right)$. $u^{a}$ defines the projection operator $h_{a b}=g_{a b}+u_{a} u_{b}$ with respect to which all geometric objects defined on $M$ can be $1+3$ decomposed.

The kinematical variables are defined by the the $1+3$ decomposition of

$$
u_{a ; b}=-\dot{u}_{a} u_{b}+\omega_{a b}+\underbrace{\sigma_{a b}+\frac{1}{3} \theta h_{a b}}_{=\theta_{a b}}
$$

$\omega_{a b}=h_{a}^{c} h_{b}^{d} u_{[c ; d]}$ is called the vorticity tensor, $\theta_{a b}=h_{a}^{c} h_{b}^{d} u_{(c ; d)}, \theta=\theta_{a}^{a}=h^{a b} u_{a ; b}=u_{; a}^{a}$ is called the expansion (isotropic strain) and $\sigma_{a b}=\theta_{a b}-\frac{1}{3} \theta h_{a b}=\left[h_{(a}^{c} h_{b)}^{d}-\frac{1}{3} h^{c d} h_{a b}\right] u_{c ; d}$ is called the shear stress tensor or simply the 
shear. From the vorticity tensor one defines the vorticity vector

$$
\omega^{a}=\frac{1}{2} \eta^{a b c d} u_{b ; c} u_{d}
$$

At the level of dynamics the $1+3$ decomposition concerns the energy momentum tensor $T_{a b}$ of a fluid in $M$ and defines the physical quantities of the fluid as observed by the observers $u^{a}$ as follows

$$
T_{a b}=\mu u_{a} u_{b}+p h_{a b}+2 q_{(a} u_{b)}+\pi_{a b} .
$$

The variables $\mu, p, q^{a}, \pi_{a b}$ have the following physical interpretation:

a. The scalars $\mu=T_{a b} u^{a} u^{b}$ and $p=\frac{1}{3} h^{a b} T_{a b}$ correspond respectively to the energy (mass) density and the isotropic pressure of the fluid.

b. The spacelike vector $q_{a}=-h_{a}^{d} T_{d c} u^{c}$ is the energy (heat) flux in the three space defined by the projection tensor $h_{a b}$.

c. $\pi_{a b}=\left(h_{a}^{c} h_{b}^{d}-\frac{1}{3} h^{c d} h_{a b}\right) T_{c d}$ is the traceless $\left(\pi_{a}^{a}=0\right)$ stress tensor (measures the anisotropy).

The fluids are classified according to the dynamical variables in dust $\left(p=q_{a}=\pi_{a b}=0\right)$, perfect fluid $\left(q_{a}=\pi_{a b}=0\right)$, heat conducting fluid $\left(q_{a} \neq 0\right)$ and anisotropic fluid $\left(\pi_{a b} \neq 0\right)$.

At the same level one considers the $1+3$ decomposition of the conservation equation $T_{; b}^{a b}=0$ which leads to the following two equations ( see for example [23])

$$
\begin{aligned}
\dot{\mu}+(\mu+p) \theta+\pi^{a b} \sigma_{a b}+q_{; a}^{a}+q^{a} \dot{u}_{a} & =0 \\
(\mu+p) \dot{u}_{a}+h_{a}^{c}\left(p_{, c}+\pi_{c ; b}^{b}+\dot{q}_{c}\right)+\left(\omega_{a c}+\sigma_{a c}+\frac{4}{3} \theta h_{a c}\right) q^{c} & =0 .
\end{aligned}
$$

Finally the $1+3$ decomposition of the Ricci identity $u_{a ; b c}-u_{a ; c b}=R_{a b c}^{d} u_{d}$ leads to two more sets of equations which are called the propagation and the constraint equations which are explicitly stated in [32].

\subsection{The $1+1+2$ decomposition}

The pair of vectors $u^{a}, n^{a}\left(n^{a} n_{a}=1, u^{a} n_{a}=0\right)$ constitutes a double congruence and defines the new projection operator

$$
p_{a b}=h_{a b}-n_{a} n_{b}
$$

which projects normal to both vectors $u^{a}, n^{a}$ on the 2 -dimensional space called the screen space.

The $1+1+2$ decomposition defines the "kinematic" variables of the spacelike vector field $n^{a}$ (see [21, 23] and references cited therein):

$$
n_{a ; b}=A_{a b}+\stackrel{*}{n}_{a} n_{b}-\dot{n}_{a} u_{b}+u_{a}\left[n^{c} u_{c ; b}+\left(n^{c} \dot{u}_{c}\right) u_{b}-\left(n^{c} u_{c}^{*}\right) n_{b}\right]
$$

where $\dot{s} \equiv s_{\ldots ; a} u^{a}, \stackrel{*}{s} \equiv s_{\ldots ; a} n^{a}$ and $A_{a b}=p_{a}^{c} p_{b}^{d} n_{c ; d}$. We decompose the screen tensor $A_{a b}$ into its irreducible parts (the kinematic variables of $n^{a}$ ):

$$
A_{a b}=\mathcal{S}_{a b}+\mathcal{R}_{a b}+\frac{1}{2} \mathcal{E}_{a b}
$$

where $\mathcal{S}_{a b}=\mathcal{S}_{b a}, \mathcal{S}_{b}^{b}=0$ is the traceless part (screen shear), $\mathcal{R}_{a b}=-\mathcal{R}_{b a}$ is the antisymmetric part (screen rotation) and $\mathcal{E}$ is the trace (screen expansion). We have the defining relations:

$$
\begin{aligned}
\mathcal{S}_{a b} & =\left(p_{a}^{c} p_{b}^{d}-\frac{1}{2} p^{c d} p_{a b}\right) n_{(c ; d)} \\
\mathcal{R}_{a b} & =p_{a}^{c} p_{b}^{d} n_{[c ; d]} \\
\mathcal{E} & =p^{c d} n_{c ; d}=n^{c} ; c+\dot{n}^{c} u_{c} .
\end{aligned}
$$

One can define also the screen rotation vector $\mathcal{R}^{a}=\frac{1}{2} \eta^{a b c d} \mathcal{R}_{b c} u_{d}$.

The $u^{a}$-term in (9) can be written:

$$
-N_{b}+2 \omega_{c b} n^{c}+p_{b}^{c} \dot{n}_{c}
$$


where

$$
N_{b}=p_{b c}\left(\dot{n}^{c}-u^{*}\right)=p_{b c} L_{\mathbf{u}} n^{c}
$$

is called the Greenberg vector. This vector is important because it vanishes iff the vector fields $u^{a}, n^{a}$ are surface forming (that is iff $L_{\mathbf{u}} n^{b}=A u^{b}+B n^{b}$ ). From the kinematics point of view the vector $N_{a}$ vanishes iff the vector field $n^{a}$ is "frozen" along the observers $u^{a}$.

Using the Greenberg vector, identity (9) is written:

$$
n_{a ; b}=A_{a b}+\stackrel{*}{n}_{a} n_{b}-\dot{n}_{a} u_{b}+u_{a} p_{b}^{c}\left(\dot{n}_{c}+2 \omega_{d c} n^{d}-N_{c}\right) .
$$

Concerning the physical variables the energy momentum tensor is $1+1+2$ decomposed as follows

$$
\begin{aligned}
T_{a b} & =\mu u_{a} u_{b}+2 \kappa u_{(a} n_{b)}+2 Q_{(a} u_{b)}+\gamma n_{a} n_{b}+2 P_{(a} n_{b)}+\frac{1}{2} \alpha p_{a b}+D_{a b} \\
& =\mu u_{a} u_{b}+p h_{a b}+2 \kappa u_{(a} n_{b)}+2 Q_{(a} u_{b)}+\bar{\gamma}\left(n_{a} n_{b}-\frac{1}{2} p_{a b}\right)+2 P_{(a} n_{b)}+D_{a b}
\end{aligned}
$$

which introduces the new dynamical variables $\kappa, \gamma, \alpha, \bar{\gamma}, Q_{a}, P_{a}, D_{a b}$ which correspond to the physical variables observed by the observers $u^{a}$. They are given by the formulae

$$
\begin{aligned}
\kappa & =-T_{a b} u^{a} n^{b} \\
\gamma & =T_{a b} n^{a} n^{b}, \quad \bar{\gamma}=\pi_{a b} n^{a} n^{b}=\gamma-p \\
\alpha & =p^{a b} T_{a b}=3 p-\gamma \\
Q_{a} & =-T_{b c} u^{b} p_{a}^{c}, \quad P_{a}=T_{b c} n^{b} p_{a}^{c}, \quad q_{a}=\kappa n_{a}+Q_{a} \\
D_{a b} & =\left(p_{a}^{c} p_{b}^{d}-\frac{1}{2} p^{c d} p_{a b}\right) T_{c d} .
\end{aligned}
$$

The physical meaning of each of the new physical variables is the following

a. The scalar $\kappa$ and the screen vector $Q_{a}$ are related to the heat conduction of the fluid

b. The scalar $\gamma$, the screen vector $P_{a}$, and the traceless screen tensor $D_{a b}$ have to do with the anisotropy of the fluid.

The $1+1+2$ dynamical variables are related to the $1+3$ dynamical variables as follows

$$
\begin{aligned}
q^{a} & =\kappa n^{a}+Q^{a} \\
\pi_{a b} & =\bar{\gamma}\left(n_{a} n_{b}-\frac{1}{2} p_{a b}\right)+2 P_{(a} n_{b)}+D_{a b} .
\end{aligned}
$$

The above decompositions are general and hold for all fluids.

\subsection{The string fluid defined by the electromagnetic field}

The energy momentum tensor (11) can be rewritten as:

$$
T_{a b}=\mu u_{a} u_{b}+\frac{1}{3}\left(p_{\|}+2 p_{\perp}\right) h_{a b}+\left(p_{\perp}-p_{\|}\right)\left(\frac{1}{3} h_{a b}-n_{a} n_{b}\right) .
$$

from which follows

$$
\begin{aligned}
p & =\frac{1}{3}\left(p_{\|}+2 p_{\perp}\right) \\
\pi_{a b} & =\left(p_{\perp}-p_{\|}\right)\left(\frac{1}{3} h_{a b}-n_{a} n_{b}\right), \\
q_{a} & =0 .
\end{aligned}
$$

It follows from (23) that (11) corresponds to an anisotropic fluid with vanishing heat flux. Furthermore we note that $n^{a}$ is an eigenvector of the anisotropic stress tensor $\pi_{a b}$ with eigenvalue $-\frac{2}{3}\left(p_{\perp}-p_{\|}\right)$. We assume 
$p_{\perp}-p_{\|} \neq 0$ otherwise the string fluid reduces to a perfect fluid with energy momentum tensor $T_{a b}=p_{\perp} g_{a b}$ which has the unphysical equation of state $\mu+p_{\perp}=0$.

Furthermore, in the $1+1+2$ decomposition the tensor $\pi_{a b}$ is written as

$$
\pi_{a b}=-\frac{2}{3}\left(p_{\perp}-p_{\|}\right)\left(n_{a} n_{b}-\frac{1}{2} p_{a b}\right),
$$

from which follows that the only nonvanishing irreducible part of $\pi_{a b}$ is:

$$
\bar{\gamma}=-\frac{2}{3}\left(p_{\perp}-p_{\|}\right) .
$$

Hence, we conclude that the string fluid defined by (23) is the "simplest" anisotropic fluid.

An important example of a string fluid is the electromagnetic field in the RMHD approximation with infinite conductivity and vanishing electric field(see [12, [13,, 14$]$ ).

Indeed in this approximation the electromagnetic tensor $F_{a b}$ is given by the expression

$$
F_{a b}=\eta_{a b c d} u^{c} H^{d}
$$

where $H^{a}$ is the magnetic field and the vector $n^{a}=H^{a} / H$ is the unit vector in the direction of the magnetic field.

The Minkowski energy momentum tensor of the electromagnetic field $T_{E M}^{a b}$ is given by

$$
{ }_{E M} T^{a b}=\lambda\left(F^{a c} F_{c}^{b}-\frac{1}{4} g^{a b} F_{c d} F^{c d}\right)
$$

where $\lambda$ is a constant. Using Maxwell equations one shows that

$$
E M T_{; b}^{a b}=-F^{a b} J_{b} .
$$

Replacing in (30) $F_{a b}$ from (29) we find

$$
{ }_{E M} T_{a b}=\frac{1}{2} \lambda H^{2} u_{a} u_{b}+\frac{1}{6} \lambda H^{2} h_{a b}+\lambda H^{2}\left(\frac{1}{3} h_{a b}-n_{a} n_{b}\right) .
$$

Replacing $h_{a b}=p_{a b}+n_{a} n_{b}$ we find

$$
{ }_{E M} T_{a b}=\frac{1}{2} \lambda H^{2}\left(u_{a} u_{b}-n_{a} n_{b}\right)+\frac{1}{2} \lambda H^{2} p_{a b}
$$

which defines an anisotropic fluid with energy momentum tensor (1) with $-\mu=p_{\|}=-p_{\perp}=-\frac{1}{2} \lambda H^{2}$. An anisotropic fluid with energy momentum tensor of the form (10) such that $-\mu=p_{\|}$we shall call perfect string fluid. From (24) - (26) follows that for a perfect fluid

$$
\begin{aligned}
\mu & =\frac{1}{2} \lambda H^{2}, \\
p & =\frac{1}{6} \lambda H^{2}, \\
\pi_{a b} & =\lambda H^{2}\left(\frac{1}{3} h_{a b}-n_{a} n_{b}\right), \\
q^{a} & =0
\end{aligned}
$$

Therefore, the equation of state parameter for the EMSF is always $p=\frac{1}{3} \mu$, and while $\mu \geq 0$, then necessarily $p \geq 0$ and the EMSF satisfies the strong energy condition. 


\section{The electromagnetic string fluid (EMSF)}

We consider the dynamical system consisting of a charged perfect fluid with isotropic pressure $p$ and energy density $\rho$ which interacts with the electromagnetic field in the RMHD approximation with infinite conductivity and vanishing electric field. Physically this situation is considered to be the case in various plasmas [31].

Due to the interaction of the fluid with the electromagnetic filed it is possible that the magnetic field produces a different fluid pressure perpendicular and parallel to the magnetic field therefore the perfect fluid becomes an anisotropic fluid with pressure distribution $p_{\|} n_{a} n_{b}+p_{\perp} p_{a b}$. The energy momentum tensor of the interacting fluid is then

$$
T_{a b}=\left(\rho+\frac{1}{2} \lambda H^{2}\right) u_{a} u_{b}+\left(p_{\|}-\frac{1}{2} \lambda H^{2}\right) n_{a} n_{b}+\left(p_{\perp}+\frac{1}{2} \lambda H^{2}\right) p_{a b} .
$$

The interacting fluid is not a perfect string fluid. For this to be the case the following condition must be satisfied

$$
\rho+\frac{1}{2} \lambda H^{2}=-\left(p_{\|}-\frac{1}{2} \lambda H^{2}\right) \Longrightarrow \rho=-p_{\|} .
$$

With this condition assumed the energy momentum of the EMSF is

$$
T_{a b}=\left(\rho+\frac{1}{2} \lambda H^{2}\right) u_{a} u_{b}-\left(\rho+\frac{1}{2} \lambda H^{2}\right) n_{a} n_{b}+\underbrace{\left(p_{\perp}+\frac{1}{2} \lambda H^{2}\right)}_{\equiv q} p_{a b} .
$$

Then $1+3$ decomposition gives

$$
\begin{aligned}
\mu & =\rho+\frac{1}{2} \lambda H^{2} \\
p & =\frac{1}{3}\left(2 p_{\perp}-\rho+\frac{1}{2} \lambda H^{2}\right) \\
q^{a} & =0 \\
\bar{\pi}_{a b} & =\left(\rho+p_{\perp}+\lambda H^{2}\right)\left(\frac{1}{3} h_{a b}-n_{a} n_{b}\right)
\end{aligned}
$$

Concerning the $1+1+2$ dynamical variables we have that for the EMSF

$$
\mu=\rho+\frac{1}{2} \lambda H^{2}, \kappa=0, Q_{a}=0, \gamma=-\rho-\frac{1}{2} \lambda H^{2}, \quad P_{a}=0, \alpha=2 p_{\perp}+\lambda H^{2}, \quad D^{a b}=0 .
$$

But

$$
\alpha=3 p-\gamma \Longrightarrow p=\frac{2}{3}\left(p_{\perp}+\frac{1}{2} \lambda H^{2}\right)+\frac{\gamma}{3}
$$

therefore

$$
\bar{\gamma}=\gamma-p \Longrightarrow \bar{\gamma}=-\frac{2}{3}\left(\rho+p_{\perp}+\lambda H^{2}\right)
$$

Finally we note the relation

$$
\mu+q=\rho+p_{\perp}+\lambda H^{2}
$$

which is useful in the calculations. Here $q=p_{\perp}+\frac{1}{2} \lambda H^{2}$ and thus for the EMSF the energy momentum tensor is written $T_{a b}=\mu\left(u_{a} u_{b}-n_{a} n_{b}\right)+q p_{a b}$ which is the general form for any perfect string fluid.

One direction in which the string fluids have been studied is the simplification of the field equations for various types of collineations of spacetime [15], [16], [18, [17, [19], 20] [29]. In the next sections we extend these studies to the case of the EMSF. 


\subsection{The Ricci tensor of the EMSF}

We consider Einstein field equations in the form $R_{a b}=T_{a b}+\left(\Lambda-\frac{1}{2} T\right) g_{a b}$ where $T \equiv T_{a}^{a}$ and compute $R_{a b}$ in terms of the string fluid variables: We find:

$$
R_{a b}=\left(p_{\perp}+\frac{1}{2} \lambda H^{2}-\Lambda\right)\left(u_{a} u_{b}-n_{a} n_{b}\right)+\left(\rho+\frac{1}{2} \lambda H^{2}+\Lambda\right) p_{a b} .
$$

We note immediately that $R_{a b}$ is found from $T_{a b}$ given by (1) for $p_{\|}=-\mu$ if we interchange $\mu \leftrightarrow p_{\perp}+\frac{1}{2} \lambda H^{2}-$ $\Lambda, \quad p_{\perp} \leftrightarrow \rho+\frac{1}{2} \lambda H^{2}+\Lambda$ and vice versa. This is a useful observation because it allows us to compute various results for $R_{a b} / T_{a b}$ and write down the answer for the corresponding quantities for $T_{a b} / R_{a b}$ by interchanging the string variables as indicated above. For example the $1+3$ decomposition of $R_{a b}$ is written directly from (23) as follows:

$$
R_{a b}=\left(p_{\perp}+\frac{1}{2} \lambda H^{2}-\Lambda\right) u_{a} u_{b}+\frac{1}{3}\left(2 \rho-p_{\perp}+\frac{1}{2} \lambda H^{2}+3 \Lambda\right) h_{a b}+\left(\rho+p_{\perp}+\lambda H^{2}\right)\left(\frac{1}{3} h_{a b}-n_{a} n_{b}\right) .
$$

\subsection{The conservation equations for the EMSF in the $1+1+2$ decomposition}

In the case of the EMSF the conservation equations (7), (8) are simplified as follows

$$
\begin{aligned}
\dot{\mu}+(\mu+q)\left(\frac{2}{3} \theta-\sigma_{a b} n^{a} n^{b}\right) & =0 \\
(\mu+q)\left[\dot{u}_{a}-\left(\mathcal{E}-\dot{n}_{b} u^{b}\right) n_{a}-h_{a}^{b} n_{b}^{*}\right]+p_{a}^{b} q_{, b}-\stackrel{*}{\mu} n_{a} & =0 .
\end{aligned}
$$

Furthermore, by projecting the second equation along $n^{a}$ and using of the tensor $p_{b}^{a}$, we get the two equations

$$
\begin{aligned}
\stackrel{*}{\mu}+(\mu+q) \mathcal{E} & =0 \\
p_{a}^{b}\left[q_{, b}+(\mu+q)\left(\dot{u}_{b}-\stackrel{*}{n}_{b}\right)\right] & =0 .
\end{aligned}
$$

Replacing the energy density $\mu$ and the heat coefficient $q$ from the expressions of the previous section we find that the $1+1+2$ decomposition of the conservation equation for an EMSF are

$$
\begin{aligned}
\dot{\rho}+\lambda H \dot{H}+\left(\rho+p_{\perp}+\lambda H^{2}\right)\left(\frac{2}{3} \theta-\sigma_{a b} n^{a} n^{b}\right) & =0 \\
\stackrel{*}{\rho}+\lambda H \stackrel{*}{H}+\left(\rho+p_{\perp}+\lambda H^{2}\right) \mathcal{E} & =0 \\
p_{a}^{b}\left[p_{\perp, b}+\lambda H H_{, b}+\left(\rho+p_{\perp}+\lambda H^{2}\right)\left(\dot{u}_{b}-\stackrel{*}{n}_{b}\right)\right] & =0 .
\end{aligned}
$$

These equations are independent of any other assumptions which one might do concerning the fluid, including the symmetries. Therefore for each additional assumption (including the symmetry assumptions) the conservation equations take a different form and in that form supplement the rest of the field equations as constraint equations.

We continue our analysis with the $1+1+2$ decomposition of Maxwell equations.

\subsection{Maxwell equations in the $1+3$ and the $1+1+2$ formalisms}

Maxwell equations are

$$
F_{[a b ; c]}=0, F_{; b}^{a b}=J^{a}
$$

where $F^{a b}$ is the electromagnetic field tensor and $J^{a}$ is the 4-current. The 4-current and the electromagnetic field tensor in the $1+3$ decomposition are decomposed as follows

$$
\begin{aligned}
J^{a} & =e u^{a}+j^{a} \\
F^{a b} & =u^{a} E^{b}-u^{b} E^{a}+\eta^{a b c d} H_{c} u_{d}
\end{aligned}
$$


where $\eta^{a b c d}$ is the alternating tensol 11 and the various physical quantities introduced are (a) $e$ the charge density (b) $j^{a}$ the conduction current (c) $E^{a}$ the electric field and (d) $H^{a}$ the magnetic field, all these quantities measured by the observer $u^{a}$. Inverting (55), (56) we find

$$
\begin{aligned}
e & =-u^{a} J_{a}, j^{a}=h_{b}^{a} J^{b} \\
E^{a} & =F^{a b} u_{b}, H^{a}=\frac{1}{2} \eta^{a b c d} F_{b c} u_{d} .
\end{aligned}
$$

At this point we would like to note that from the from the $1+3$ decomposition of $F_{a b}$ wrt $u^{a}$ it follows that the magnetic part $H^{a}$ is orthogonal to $u^{a}$, i.e. $H^{a} u_{a}=0$. Thereupon, the chosen direction $n^{a}=H^{a} / H$ satisfies the relation $n^{a} u_{a}=0$. That's why the direction of the magnetic field wrt the observer $u^{a}$ is always taken orthogonal.

Taking into account the $1+3$ kinematic variables Maxwell equations are $1+3$ decomposed wrt the observer $u^{a}$ into the following constraint and propagation equations (see [32, 34])

$$
\begin{aligned}
h_{b}^{a} H_{; a}^{b} & =2 \omega^{a} E_{a} \\
h_{b}^{a} E_{; a}^{b} & =e-2 \omega^{a} H_{a} \\
h_{b}^{a} \dot{H}^{b} & =u_{; b}^{a} H^{b}-\theta H^{a}-I^{a}(E) \\
h_{b}^{a} \dot{E}^{b} & =u_{; b}^{a} E^{b}-\theta E^{a}+I^{a}(H)-j^{a}
\end{aligned}
$$

where:

$$
\begin{aligned}
I^{a}(E) & =\eta^{a b c d} u_{b}\left(\dot{u}_{c} E_{d}-E_{c ; d}\right) \\
I^{a}(H) & =\eta^{a b c d} u_{b}\left(\dot{u}_{c} H_{d}-H_{c ; d}\right)
\end{aligned}
$$

and $\omega^{a}=\frac{1}{2} \eta^{a b c d} u_{b ; c} u_{d}$ and $\theta=u_{; a}^{a}$ are the the vorticity vector and the expansion of the fluid as measured by the observers $u^{a}$. A dot over a symbol denotes covariant differentiation wrt $u^{a}$ (i.e. along the fluid particle world line).

If we operate on (63) and (64) with $\eta^{a b c d} u_{d}$ then a direct calculation yields the following two mathematical identities:

$$
\begin{aligned}
& E_{[r ; s]}=u_{[r} \dot{E}_{s]}+\dot{u}_{[r} E_{s]}+u^{t} E_{t ;[r} u_{s]}+\frac{1}{2} \eta_{r s t m} u^{t} I^{m}(E) \\
& H_{[r ; s]}=u_{[r} \dot{H}_{s]}+\dot{u}_{[r} H_{s]}+u^{t} H_{t ;[r} u_{s]}+\frac{1}{2} \eta_{r s t m} u^{t} I^{m}(H) .
\end{aligned}
$$

From the identity (66) one computes the screen rotation of the magnetic field lines. The result is:

$$
\begin{aligned}
\mathcal{R}_{a b} & =p_{a}^{c} p_{b}^{d} n_{[c ; d]}=\frac{1}{H} p_{a}^{c} p_{b}^{d} H_{[c ; d]} \\
& =-\frac{1}{2 H} p_{a}^{c} p_{b}^{d} \eta_{c d r s} I^{r}(H) u^{s} .
\end{aligned}
$$

\footnotetext{
${ }^{1}$ In Minkowski spacetime the alternating tensor is defined as follows:

$$
\eta^{a b c d}=\eta^{[a b c d]}, \eta^{0123}=(-g)^{-1 / 2}
$$

where $g=\operatorname{det}\left(g_{a b}\right)$.It satisfies the properties:

$$
\eta^{a b c d} \eta_{\text {arst }}=-3 ! \delta_{r}^{[b} \delta_{s}^{c} \delta_{t}^{d]}, \eta^{a b c d} \eta_{a b s t}=-4 \delta_{s}^{[c} \delta_{t}^{d]}
$$
}

In the Euclidian 3-d space the alternating tensor is defined as follows:

$$
\eta^{\mu \nu \rho}=\eta^{[\mu \nu \rho]}, \eta^{123}=h^{-1 / 2}
$$

where $h=\operatorname{det}\left(h_{\mu \nu}\right)$. It satisfies the properties:

$$
\eta^{\mu \nu \rho} \eta_{\mu \sigma \tau}=2 \delta_{\sigma}^{[\nu} \delta_{\tau}^{\rho]}, \eta^{\mu \nu \rho} \eta_{\mu \nu \tau}=2 \delta_{\tau}^{\rho} .
$$


Proposition 1 The screen rotation vector of the magnetic field lines is proportional to the magnetic field as follows:

$$
\mathcal{R}^{a}=-\frac{H_{c} I^{c}(H)}{2 H^{3}} H^{a}
$$

Proof

Expanding $p_{a}^{c}, p_{b}^{d}$ in (67) we get:

$$
\mathcal{R}_{a b}=-\frac{1}{2 H} \eta_{a b r s} I^{r}(H) u^{s}-\frac{1}{H^{3}} H_{[a} \eta_{b] c r s} H^{c} I^{r}(H) u^{s}
$$

We operate with $\eta^{a b p q}$ on both sides and find:

$$
\begin{aligned}
-\frac{1}{2 H} \eta^{a b p q} \eta_{a b r s} I^{r}(H) u^{s} & =\frac{1}{H}\left[I^{p}(H) u^{q}-I^{q}(H) u^{p}\right] \\
-\frac{1}{H^{3}} \eta^{a b p q} H_{[a} \eta_{b] c r s} H^{c} I^{r}(H) u^{s} & =\frac{1}{H}\left[-I^{p}(H) u^{q}+I^{q}(H) u^{p}\right]+\frac{H_{c} I^{c}(H)}{H^{3}}\left[H^{p} u^{q}-H^{q} u^{p}\right] .
\end{aligned}
$$

Thus

$$
\eta^{a b p q} \mathcal{R}_{a b}=\frac{H_{c} I^{c}(H)}{H^{3}}\left[H^{p} u^{q}-H^{q} u^{p}\right] .
$$

In terms of the screen rotation vector $\mathcal{R}^{a}=\frac{1}{2} \eta^{a b c d} u_{b} \mathcal{R}_{c d}$ equation (69) is written:

$$
\mathcal{R}^{a}=-\frac{H_{c} I^{c}(H)}{2 H^{3}} H^{a}
$$

which completes the proof.

From Proposition 1 we infer that the screen rotation of the magnetic field congruence vanishes iff $H_{c} I^{c}(H)$ $=0$.

\subsection{Maxwell equations in the RMHD approximation}

In the RMHD approximation with infinite electric conductivity and vanishing electric field Maxwell equations become:

$$
\begin{aligned}
h_{b}^{a} H_{; a}^{b} & =0 \\
e & =2 \omega^{a} H_{a} \\
h_{b}^{a} \dot{H}^{b} & =u_{; b}^{a} H^{b}-\theta H^{a} \\
I^{a}(H) & =j^{a} .
\end{aligned}
$$

Let $n^{a}=H^{a} / H$ be the unit vector in the direction of the magnetic field. Geometrically $n^{a}$ is the unit tangent to the spacelike magnetic field lines. The pair $\left(u^{a}, n^{a}\right)$ forms a double congruence. Maxwell equations in terms of the irreducible parts defined by this double congruence take a geometric form. The constraint equation (70) for the magnetic field gives:

$$
H h_{b}^{a} n_{; a}^{b}+H_{, a} n^{a}=0
$$

But $h_{b}^{a} n_{; a}^{b}=p_{b}^{a} n_{; a}^{b}=\mathcal{E}$ where $\mathcal{E}$ is the screen expansion of the magnetic field lines. Therefore

$$
\mathcal{E}=-(\ln H)^{*}
$$

where a "*" over a symbol means covariant differentiation wrt $n^{a}$. From this equation we infer that the stronger the magnetic field the denser the magnetic field lines on the screen space, that is the greater is the magnetic flux through the screen space (as expected).

We examine now the propagation equation (72) of the magnetic field. We have:

$$
\frac{\dot{H}}{H} n^{a}+h_{b}^{a} \dot{n}^{b}=u_{; b}^{a} n^{b}-\theta n^{a} .
$$


Contracting with $n^{a}$ and projecting with $p_{b}^{a}$ we get the pair of equations:

$$
\begin{aligned}
(\ln H)^{\cdot} & =\sigma_{a b} n^{a} n^{b}-\frac{2}{3} \theta \\
N^{a} & \equiv p_{b}^{a} \mathcal{L}_{u} n^{b}=0 .
\end{aligned}
$$

The first equation involves the change of the strength of the magnetic field along the flow lines of the fluid i.e. the field $u^{a}$. A kinematic interpretation is that the vector $n^{a}$ is an eigenvector of the shear with eigenvalue $(\ln H)^{\cdot}+\frac{2}{3} \theta$.

The second is the geometric condition that the magnetic field lines are material lines in the fluid and correspond to the statement that the magnetic field is "frozen" along the fluid. Physically this means that each particle of the fluid moves always on the same magnetic field line.

Relation (73) due to (68) it is written as:

$$
\mathcal{R}^{a}=-\frac{H_{c} j^{c}}{2 H^{3}} H^{a}
$$

therefore in the RMHD approximation the screen rotation of the magnetic field lines vanishes iff the conduction current $j^{a}$ is normal to the magnetic field.

Ohm's Law in its generalized form which includes the Hall current is written (see [30]):

$$
J^{a}=\rho u^{a}+\frac{1}{\left(1+\lambda^{2} B^{c} B_{c}\right)}\left[k E^{a}+\lambda k \eta^{a b c d} E_{b} u_{c} B_{d}+\lambda^{2} k\left(E^{c} B_{c}\right) B^{a}\right] .
$$

In the RMHD approximation we have that the spatial part $j^{a}$ of the 4 -current $J^{a}$ vanishes, therefore $\mathcal{R}^{a}=0$. Hence in a perfectly conducting fluid for which generalized Ohm's law applies, the magnetic field lines have zero rotation as measured by $u^{a}$. Because (78) is not the most general form of Ohm's Law we shall assume in the following $\mathcal{R}^{a}$ to be given by (77).

Summarizing we have that in the RMHD approximation Maxwell equations are:

$$
\begin{aligned}
\mathcal{E} & =-(\ln H)^{*} \\
\mathcal{R}^{a} & =-\frac{H_{c} j^{c}}{2 H^{3}} H^{a} \\
e & =2 \omega^{a} H_{a} \\
(\ln H)^{\cdot} & =\sigma_{a b} n^{a} n^{b}-\frac{2}{3} \theta \\
N^{a} & \equiv p_{b}^{a} \mathcal{L}_{u} n^{b}=0 .
\end{aligned}
$$

Note that equation (83) can be written in the equivalent form:

$$
p_{b}^{a} \dot{n}^{b}=\left(p_{. c}^{a} \sigma_{b}^{c}+\omega_{. b}^{a}\right) n^{b} .
$$

These equations are general and independent of further simplifying assumptions (e.g. symmetry assumptions) we might do, and hold in all cases.

\section{The field equations for the EMSF}

The EMSF must satisfy three sets of equations: a) Maxwell equations, b) Conservation laws and c) Einstein field equations. We have already given Maxwell equations and the conservation equations.

Concerning Einstein field equations we shall consider their Lie derivative along some characteristic direction of the EM fluid. The reason for this is that we want to employ symmetry assumptions, that is equations of the form $\mathcal{L}_{\xi} M_{a b}=A_{a b}$ where $M_{a b}$ is a tensor computed in terms of the metric (or the metric itself) and $A_{a b}$ is an arbitrary tensor having the same symmetries as the $M_{a b}$. Due to the form of Einstein field equations we compute $\mathcal{L}_{\xi} R_{a b}$ in terms of $\mathcal{L}_{\xi} g_{a b}$ using various identities of Riemannian Geometry. Then we impose the symmetry assumption by choosing a specific form for $A_{a b}$. For example for a CKV $M_{a b}=g_{a b}$ and $A_{a b}=2 \psi g_{a b}$ 
where $\psi\left(x^{a}\right)$ is the conformal factor. Then we replace $\mathcal{L}_{\xi} R_{a b}$ in the Lie derivative of the field equations and we find the field equations in a form that incorporates already the imposed geometric symmetry assumption.

In a previous work on string fluids 29] we have computed Einstein equations for a perfect string fluid and many types of symmetries in the cases that the symmetry vector is either $\xi^{a}=\xi u^{a}$ or $\xi^{a}=\xi n^{a}$. Therefore we could write straight away the field equations in the case of an EMSF by simply specifying $n^{a}=H^{a} / H$. Of course in this case the resulting equations will be supplemented by Maxwell equations. In the following we recall briefly some important intermediate steps in order to make the present work more readable and self contained. Details can be found in 29 .

The Lie derivative of the Ricci tensor wrt a general time-like vector $\xi^{a}=\xi u^{a}$ has been computed (see equation (3.9) in [23]) in terms of the standard dynamic variables $\mu, p, q_{a}, \pi_{a b}$. By using the general expression, $L_{\xi} R_{a b}$ can be written in terms of the perfect string fluid parameters $\rho, q$. In a similar way, the Lie derivative of the Ricci tensor along the spacelike vector $\xi^{a}=\xi n^{a}$ can be expressed in terms of the $1+1+2$ dynamic quantities.

Using Maxwell equations we show easily that the conservation equations (51), (52) and (53) (which must also be satisfied in all cases) are simplified as follows:

$$
\begin{aligned}
\dot{\rho}-\left(\rho+p_{\perp}\right)(\ln H)^{\cdot} & =0 \\
\stackrel{*}{\rho}-\left(\rho+p_{\perp}\right)(\ln H)^{*} & =0 \\
p_{a}^{b}\left[p_{\perp, b}+\lambda H H_{, b}+\left(\rho+p_{\perp}+\lambda H^{2}\right)\left(\dot{u}_{b}-\stackrel{*}{n}_{b}\right)\right] & =0 .
\end{aligned}
$$

Concerning the Einstein filed equations we have from 23] and [29] the results:

$$
\begin{aligned}
& \frac{1}{\xi} L_{\xi} R_{a b}=\left[\dot{q}+2(q-\Lambda)(\ln \xi)^{\cdot}\right] u_{a} u_{b}+2(q-\Lambda)\left[\dot{u}_{c}-(\ln \xi)_{, c}\right] u_{(a} h_{b)}^{c}+ \\
& +\frac{1}{3}\left[2 \dot{\mu}-\dot{q}+\frac{2}{3}(2 \mu-q+3 \Lambda) \theta\right] h_{a b}+ \\
& +\left[\dot{\mu}+\dot{q}+\frac{2}{3}(\mu+q) \theta\right]\left(\frac{1}{3} h_{a b}-n_{a} n_{b}\right)+\frac{2}{3}(2 \mu-q+3 \Lambda) \sigma_{a b}+ \\
& +2(\mu+q)\left(\frac{1}{3} h_{c d}-n_{c} n_{d}\right) \delta_{(a}^{d}\left(\omega_{. b)}^{c}+\sigma_{. b)}^{c}\right)- \\
& -2(\mu+q) \dot{n}_{d} h_{(a}^{d} n_{b)} \text {. } \\
& \frac{1}{\xi} L_{\xi} R_{a b}=\left[\stackrel{*}{q}+2(q-\Lambda) \dot{u}_{c} n^{c}\right] u_{a} u_{b}-2(q-\Lambda)\left[\stackrel{*}{u}_{c} n_{c}-(\ln \xi)^{\cdot}\right] u_{(a} n_{b)}- \\
& -\left[\stackrel{*}{q}+2(q-\Lambda)(\ln \xi)^{*}\right] n_{a} n_{b}- \\
& -2\left[(\mu+\Lambda) N_{c}+2(q-\Lambda) \omega_{d c} n^{d}\right] u_{(a} p_{b)}^{c}- \\
& -2(q-\Lambda) p_{c}^{d}\left[\stackrel{*}{n}_{d}+(\ln \xi)_{, d}\right] n_{(a} p_{b)}^{c}+[\stackrel{*}{\mu}+(\mu+\Lambda) \mathcal{E}] p_{a b}+ \\
& +2(\mu+\Lambda) \mathcal{S}_{a b} \text {. }
\end{aligned}
$$

Expressions (88) and (89) are general and hold for all collineations and all perfect string fluids. For each type of collineation the lhs of the expressions (88) and (89) simplifies accordingly and for each specific perfect string fluid the rhs is simplified the same. Equating the two parts one finds immediately Einstein field equations for the specific string fluid considered and the specific symmetry assumed.

In the case of the EMSF we have $\mu=\rho+\frac{1}{2} \lambda H^{2}$ and $q=p_{\perp}+\frac{1}{2} \lambda H^{2}$. The result applies to all collineations concerning the EMSF.

For easy reference we collect below the results of the calculations :

Maxwell equations: 


$$
\begin{aligned}
N^{a} & =0 \Longleftrightarrow p_{b}^{a} \dot{n}^{b}=\left(p_{. c}^{a} \sigma_{b}^{c}+\omega_{. b}^{a}\right) n^{b} \\
\mathcal{E} & =-(\ln H)^{*} \\
\sigma_{a b} n^{a} n^{b}-\frac{2}{3} \theta & =(\ln H)^{\cdot} \\
e & =2 \omega^{a} H_{a}, \quad \mathcal{R}^{a}=-\frac{H_{c} j^{c}}{2 H^{3}} H^{a}
\end{aligned}
$$

Conservation equations:

$$
\begin{aligned}
\dot{\rho}-\left(\rho+p_{\perp}\right)(\ln H)^{\cdot} & =0 \\
\stackrel{*}{\rho}-\left(\rho+p_{\perp}\right)(\ln H)^{*} & =0 \\
p_{a}^{b}\left[p_{\perp, b}+\lambda H H_{, b}+\left(\rho+p_{\perp}+\lambda H^{2}\right)\left(\dot{u}_{b}-\stackrel{*}{n}_{b}\right)\right] & =0 .
\end{aligned}
$$

Einstein filed equations:

$$
\begin{aligned}
\frac{1}{\xi} L_{\xi} R_{a b}= & {\left[p_{\perp}+\lambda H \dot{H}+2\left(p_{\perp}+\frac{1}{2} \lambda H^{2}-\Lambda\right)(\ln \xi)^{\cdot}\right] u_{a} u_{b}+2\left(p_{\perp}+\frac{1}{2} \lambda H^{2}-\Lambda\right)\left[\dot{u}_{c}-(\ln \xi)_{, c}\right] u_{(a} h_{b)}^{c}+} \\
& +\frac{1}{3}\left[2 \dot{\rho}-\dot{p}_{\perp}+\lambda H \dot{H}+\frac{2}{3}\left(2 \rho-p_{\perp}+\frac{1}{2} \lambda H^{2}+3 \Lambda\right) \theta\right] h_{a b}+ \\
& +\left[\dot{\rho}+\dot{p}_{\perp}+2 \lambda H \dot{H}+\frac{2}{3}\left(\rho+p_{\perp}+\lambda H^{2}\right) \theta\right]\left(\frac{1}{3} h_{a b}-n_{a} n_{b}\right)+\frac{2}{3}\left(2 \rho-p_{\perp}+\frac{1}{2} \lambda H^{2}+3 \Lambda\right) \sigma_{a b}+ \\
& +2\left(\rho+p_{\perp}+\lambda H^{2}\right)\left(\frac{1}{3} h_{c d}-n_{c} n_{d}\right) \delta_{(a}^{d}\left(\omega_{. b)}^{c}+\sigma_{. b)}^{c}\right)- \\
& -2\left(\rho+p_{\perp}+\lambda H^{2}\right) \dot{n}_{d} h_{(a}^{d} n_{b)} . \\
\frac{1}{\xi} L_{\xi} R_{a b}= & {\left[p_{\perp}^{*}+\lambda H \stackrel{*}{H}+2\left(p_{\perp}+\frac{1}{2} \lambda H^{2}-\Lambda\right) \dot{u}_{c} n^{c}\right] u_{a} u_{b}-2\left(p_{\perp}+\frac{1}{2} \lambda H^{2}-\Lambda\right)\left[\stackrel{*}{u}_{c} n^{c}-(\ln \xi)^{\cdot}\right] u_{(a} n_{b)}-} \\
& -\left[p_{\perp}^{*}+\lambda H \stackrel{*}{H}+2\left(p_{\perp}+\frac{1}{2} \lambda H^{2}-\Lambda\right)(\ln \xi)^{*}\right] n_{a} n_{b}- \\
& -4\left(p_{\perp}+\frac{1}{2} \lambda H^{2}-\Lambda\right) \omega_{d c} n^{d} u_{(a} p_{b)}^{c}- \\
& -2\left(p_{\perp}+\frac{1}{2} \lambda H^{2}-\Lambda\right) p_{c}^{d}\left[\stackrel{*}{n}_{d}+(\ln \xi)_{, d}\right] n_{(a} p_{b)}^{c}+\left[\stackrel{*}{\rho}+\lambda H \stackrel{*}{H}+\left(\rho+\frac{1}{2} \lambda H^{2}+\Lambda\right) \mathcal{E}\right] p_{a b}+ \\
& +2\left(\rho+\frac{1}{2} \lambda H^{2}+\Lambda\right) \mathcal{S}_{a b} .
\end{aligned}
$$

At this point recall that for an arbitrary vector field $\xi^{a}$ we can always select $n^{a}$ such that

$$
\xi^{a}=\xi(u) u^{a}+\xi(n) n^{a}
$$

to be always true. Hence, the Lie derivative $L_{\xi} M_{a b}$ can be always expressed as

$$
\mathcal{L}_{\xi} M_{a b}=\mathcal{L}_{\xi(u) u^{a}} M_{a b}+\mathcal{L}_{\xi(n) n^{a}} M_{a b}
$$

which means that we have two components. We continue by studying the special cases where $\xi(n)=0$ and $\xi^{a}$ is timelike, and $\xi(u)=0$ and $\xi^{a}$ is spacelike. 


\section{$5 \quad$ The EMSF admitting a timelike CKV $\xi^{a}=\xi u^{a}(\xi>0)$}

As it has mentioned there are two types of equations constraining the evolutions of a gravitational system which admits a symmetry, that is the kinematic conditions and dynamic equations.

The kinematic conditions are equations among the kinematic variables of the gravitational system which result from geometric identities and additional geometric assumptions (such as symmetries). The dynamic equations do not necessarily inherit the kinematic symmetries of the system. In the following we consider two types of symmetries (a) CKVs defined by timelike vectors $\xi^{a}=\xi u^{a}(\xi>0)$; and (b) CKVs defined by the spacelike vectors $\xi^{a}=\xi n^{a}(\xi>0)$.

\subsection{The case of a timelike $\mathrm{CKV} \xi^{a}=\xi u^{a}(\xi>0)$}

We look first on the kinematic implications of the assumed symmetry and then on the dynamical ones.

\subsection{The kinematic implications}

From previous works we have the following kinematic conditions for a $\mathrm{CKV} \xi^{a}=\xi u^{a}[24]$.

Proposition 2 A fluid space-time $u^{a}$ admits a $C K V \xi^{a}=\xi u^{a}$ iff:

1. $\sigma_{a b}=0$

2. $\dot{u}_{a}=(\ln \xi)_{, a}+\frac{1}{3} \theta u_{a}$ where $\sigma_{a b}, \theta$ and $\dot{u}^{a}$ are, respectively, the shear, expansion and acceleration of the timelike congruence generated by $u^{a}$. The conformal factor $\psi=\frac{1}{3} \xi \theta=\dot{\xi}$.

The conditions imposed by Proposition 2 supplement Maxwell equations and simplify the conservation equations. Because $\sigma_{a b}=0$ the "energy" conservation equation (85) gives:

$$
\dot{\rho}+\frac{2}{3}\left(\rho+p_{\perp}\right) \theta=0 .
$$

Equation (86) remains the same and equation (87) becomes:

$$
p_{a}^{b}\left[p_{\perp, b}+\lambda H H_{, b}+\left(\rho+p_{\perp}+\lambda H^{2}\right)\left((\ln \xi)_{, b}-\stackrel{*}{n}_{b}\right)\right]=0 .
$$

Eventually the conservation equations are equations (86), (99), (100).

\subsection{The dynamic implications}

For a CKV we have the identity:

$$
L_{\xi} R_{a b}=-2 \psi_{; a b}-g_{a b} \square \psi .
$$

The $1+3$ decomposition of $\psi_{; a b}$ wrt $u^{a}$ is (note that $\psi_{; a b}=\psi_{; b a}$ ):

$$
\psi_{; a b}=\lambda_{\psi} u_{a} u_{b}+p_{\psi} h_{a b}+2 q_{\psi(a} u_{b)}+\pi_{\psi a b}
$$

where:

$$
\lambda_{\psi}=\psi_{; a b} u^{a} u^{b}, p_{\psi}=\frac{1}{3} \psi_{; a b} h^{a b}, q_{\psi a}=-\psi_{; b c} h_{a}^{b} u^{c}, \pi_{\psi a b}=\left(h_{a}^{r} h_{b}^{s}-\frac{1}{3} h_{a b} h^{r s}\right) \psi_{; r s} .
$$

We also compute:

$$
\square \psi=\psi_{; a b} g^{a b}=-\lambda_{\psi}+3 p_{\psi} .
$$

Therefore for a $\mathrm{CKV} \xi^{a}=\xi u^{a}$ we have that:

$$
L_{\xi} R_{a b}=-\left[3\left(\lambda_{\psi}-p_{\psi}\right) u_{a} u_{b}-\left(\lambda_{\psi}-5 p_{\psi}\right) h_{a b}+4 q_{\psi(a} u_{b)}+2 \pi_{\psi a b}\right] .
$$

Using the kinematic conditions and the conservation equations, the rhs of equation (97) simplifies as follow:2

\footnotetext{
${ }^{2}$ It is easy to show (use Maxwell equations in RMHD approximation) that $\dot{n}_{d} h_{(a}^{d} n_{b)}+n_{c} n_{d} \delta_{(a}^{d} \omega_{. b)}^{c}=0$
} 


$$
\begin{aligned}
\frac{1}{\xi} L_{\xi} R_{a b}= & {\left[\dot{p}_{\perp}+\frac{1}{2} \lambda H \dot{H}+2\left(p_{\perp}-\Lambda\right) \frac{1}{3} \theta\right] u_{a} u_{b}-\frac{1}{3}\left[\dot{p}_{\perp}+2\left(p_{\perp}-\Lambda\right) \theta-\frac{1}{2} \lambda H \dot{H}\right] h_{a b} } \\
& +\left[\dot{p}_{\perp}+\lambda H \dot{H}\right]\left(\frac{1}{3} h_{a b}-n_{a} n_{b}\right) .
\end{aligned}
$$

From (104) and (105) we find that the field equations for an EM string fluid admitting the CKV $\xi^{a}=\xi u^{a}$ are

$$
\begin{aligned}
& {\left[\dot{p}_{\perp}+\frac{1}{2} \lambda H \dot{H}+2\left(p_{\perp}-\Lambda\right) \frac{1}{3} \theta\right] u_{a} u_{b}-\frac{1}{3}\left[\dot{p}_{\perp}+2\left(p_{\perp}-\Lambda\right) \theta-\frac{1}{2} \lambda H \dot{H}\right] h_{a b} } \\
& +\left[\dot{p}_{\perp}+\lambda H \dot{H}\right]\left(\frac{1}{3} h_{a b}-n_{a} n_{b}\right) \\
= & -\frac{1}{\xi}\left[3\left(\lambda_{\psi}-p_{\psi}\right) u_{a} u_{b}-\left(\lambda_{\psi}-5 p_{\psi}\right) h_{a b}+4 q_{\psi(a} u_{b)}+2 \pi_{\psi a b}\right]
\end{aligned}
$$

This relation implies the field equation: 3 :

$$
\begin{aligned}
p_{\perp}+\lambda H \dot{H} & =-\frac{3}{\xi}\left(p_{\psi}+\lambda_{\psi}\right) \\
\left(p_{\perp}+\frac{1}{2} \lambda H^{2}-\Lambda\right) \theta & =\frac{9}{\xi} p_{\psi} \\
3\left(p_{\psi}+\lambda_{\psi}\right)\left(\frac{1}{3} h_{a b}-n_{a} n_{b}\right) & =2 \pi_{\psi a b} \\
q_{\psi}^{a} & =0 .
\end{aligned}
$$

Eqn (106) using also $(\ln \xi)^{\cdot}=\frac{1}{3} \theta$ can be written :

$$
\begin{aligned}
\left(p_{\perp}+\frac{1}{2} \lambda H^{2}-\Lambda\right) & =-\left(p_{\perp}+\frac{1}{2} \lambda H^{2}-\Lambda\right)(\ln \xi)^{\cdot}-3 \lambda_{\psi} \Longrightarrow \\
\lambda_{\psi} & =-\frac{1}{3}\left[\left(p_{\perp}+\frac{1}{2} \lambda H^{2}-\Lambda\right) \xi\right]
\end{aligned}
$$

and (107):

$$
p_{\psi}=\frac{1}{3}\left(p_{\perp}+\frac{1}{2} \lambda H^{2}-\Lambda\right) \dot{\xi}
$$

The final set of equations which results from the assumption that the EMSF admits the CKV $\xi^{a}=\xi u^{a}$ is the following:

Geometric implications:

$$
\begin{aligned}
\sigma_{a b} & =0 \\
\dot{u}_{a} & =(\ln \xi)_{, a}+\frac{1}{3} \theta u_{a}
\end{aligned}
$$

\footnotetext{
${ }^{3}$ The same equations are found from $[29]$ where the field equations were:

$$
\begin{aligned}
\dot{q} & =-\frac{3}{\xi}\left(p_{\psi}+\lambda_{\psi}\right) \\
(q-\Lambda) \theta & =\frac{9}{\xi} p_{\psi} \\
3\left(p_{\psi}+\lambda_{\psi}\right)\left(\frac{1}{3} h_{a b}-n_{a} n_{b}\right) & =2 \pi_{\psi a b} . \\
q_{\psi}^{a} & =0
\end{aligned}
$$
}


Maxwell equations:

$$
\begin{aligned}
N^{a} & =0 \\
\mathcal{E} & =-(\ln H)^{*} \\
p_{b}^{a} \dot{n}^{b} & =\omega_{. b}^{a} n^{b} \\
-\frac{2}{3} \theta & =(\ln H)^{.}
\end{aligned}
$$

Conservation equations:

$$
\begin{aligned}
\dot{\rho}-\left(\rho+p_{\perp}\right)(\ln H)^{*} & =0 \\
\stackrel{*}{\rho}-\left(\rho+p_{\perp}\right)(\ln H)^{*} & =0 \\
p_{a}^{b}\left[p_{\perp, b}+\lambda H H_{, b}+\left(\rho+p_{\perp}+\lambda H^{2}\right)\left((\ln \xi)_{, b}-\stackrel{*}{n}_{b}\right)\right] & =0 .
\end{aligned}
$$

Gravitational Field equations:

$$
\begin{aligned}
\lambda_{\psi} & =-\frac{1}{3}\left[\left(p_{\perp}+\frac{1}{2} \lambda H^{2}-\Lambda\right) \xi\right] \\
p_{\psi} & =\frac{1}{3}\left(p_{\perp}+\frac{1}{2} \lambda H^{2}-\Lambda\right) \dot{\xi} \\
2 \pi_{\psi a b} & =-\xi\left(p_{\perp}+\frac{1}{2} \lambda H^{2}-\Lambda\right)\left(\frac{1}{3} h_{a b}-n_{a} n_{b}\right) \\
q_{\psi}^{a} & =0 .
\end{aligned}
$$

This is the complete set of equations which must be satisfied by the various variables (geometric, kinematic and dynamic) of an EMSF which admits the CKV $\xi^{a}=\xi u^{a}$.

\subsection{The case of an EMSF admitting a timelike CKV $\xi^{a}=\xi u^{a}$ in the FRW space- time}

We apply the results of the last section to the case of the FRW spacetime. The FRW spacetime has metric (in conformal coordinates):

$$
d s^{2}=R^{2}(\tau)\left[-d \tau^{2}+U^{2}\left(x^{\mu}\right) d \sigma_{E}^{2}\right]
$$

where the function $U^{2}\left(x^{\mu}\right)=\left(1+\frac{k}{4} \mathbf{x} \cdot \mathbf{x}\right)^{-1}$ and $k=0, \pm 1$. This metric admits the gradient CKV $\partial_{\tau}$ whose conformal factor is $\psi=\frac{d R}{d \tau}$. We define the timelike unit vector $u^{a}=\frac{1}{R} \partial_{\tau}$. If we define the new coordinate $t$ by the requirement:

$$
d \tau=\frac{1}{R(t)} d t
$$

then the metric is written:

$$
d s^{2}=-d t^{2}+R^{2}(t) U^{2}\left(x^{\mu}\right) d \sigma_{E}^{2}
$$

and the unit vector $u^{a}=\partial_{t}$. The conformal factor becomes:

$$
\psi=\frac{d R}{d t} \equiv \dot{R}(t)
$$

Then话

$$
\psi_{; a b}=\psi_{, a b}-\psi_{, c}\left\{\begin{array}{c}
c \\
a b
\end{array}\right\}=\dddot{R} \delta_{a}^{0} \delta_{b}^{0}-\psi_{, 0}\left\{\begin{array}{c}
0 \\
a b
\end{array}\right\}=\dddot{R} u_{a} u_{b}-\frac{1}{2} \ddot{R} g_{a b, 0}
$$

\footnotetext{
${ }^{4}$ In the coordinates $\{t, x, y, z\}$ we have $g_{a b}=\operatorname{diag}\left(-1, R^{2} U^{2}, R^{2} U^{2}, R^{2} U^{2}\right)$, that is, $g^{a b}=\operatorname{diag}\left(-1, \frac{1}{R^{2} U^{2}}, \frac{1}{R^{2} U^{2}}, \frac{1}{R^{2} U^{2}}\right)$, where $u^{a}=(1,0,0,0), u_{a}=(-1,0,0,0)$ and $\xi^{a}=R u^{a}$
} 
where

$$
\left\{\begin{array}{l}
0 \\
a b
\end{array}\right\}=\frac{1}{2} g^{0 c}\left(g_{a c, b}+g_{b c, a}-g_{a b, c}\right)=\frac{1}{2} g^{00}\left(g_{a 0, b}+g_{b 0, a}-g_{a b, 0}\right)=\frac{1}{2} g_{a b, 0} .
$$

For $\mu=1,2,3$ we find $\left\{\begin{array}{l}0 \\ \mu \mu\end{array}\right\}=\frac{1}{2} g_{\mu \mu, 0}=U^{2} R \dot{R}$ and $g_{\mu \mu, 0}=2 U^{2} R \dot{R}$. From $1+3$ decomposition over $\psi_{; a b}$

$$
\lambda_{\psi}=\dddot{R}, p_{\psi}=-\frac{\dot{R} \ddot{R}}{R}, q_{\psi}^{a}=0, \pi_{\psi a b}=-\frac{1}{2} \ddot{R} g_{a b, 0}+\frac{\dot{R} \ddot{R}}{R} h_{a b} .
$$

Equation (122) gives $(\dot{R} \neq 0)$

$$
\ddot{R}=-\frac{1}{3}\left(p_{\perp}+\frac{1}{2} \lambda H^{2}-\Lambda\right) R .
$$

Then $p_{\perp}+\frac{1}{2} \lambda H^{2}-\Lambda=0$ is a possible assumption which implies that $\ddot{R}=0$; thus $\psi=\dot{R}=$ const and $R(t)=b t+c$ for some real constants $b, c$.

We compute:

$$
\psi_{; a b}=\dddot{R} \delta_{a}^{t} \delta_{b}^{t}
$$

which implies:

$$
\lambda_{\psi}=\dddot{R}, p_{\psi}=0, q_{\psi a}, \pi_{\psi a b}=0 .
$$

Field equation (111) gives (as $\psi \neq$ const and $\dot{R} \neq 0$ ):

$$
p_{\perp}=-\frac{1}{2} \lambda H^{2}+\Lambda
$$

and (110) $\lambda_{\psi}=\dddot{R}=0$. It follows that:

1) $\partial_{\tau}=R(t) u^{a}$ is a special CKV or one of its specializations (If $\psi=0 \Rightarrow R(t)=$ const the spacetime reduces to a Einstein space);

2) The conformal factor is $\psi=b t+c$;

3) The spacetime admits the special gradient $\mathrm{CKV} \psi_{, a}=b \delta_{a}^{t}$;

4) The scale factor $R(t)=\frac{1}{2} b t^{2}+c t+d$.

We work now with the rest of the conservation equations. Equation (118) using $p_{\perp}=-\frac{1}{2} \lambda H^{2}+\Lambda$ gives

$$
\begin{aligned}
\dot{\mu}-\left(\mu-\frac{1}{2} \lambda H^{2}+\Lambda\right)(\ln H)^{\cdot} & =0 \Longrightarrow \dot{\mu}-(\mu+\Lambda)(\ln H)^{\cdot}+\frac{1}{2} \lambda H^{2}(\ln H)^{\cdot}=0 \Longrightarrow \\
\frac{\dot{\mu}}{\mu+\Lambda}-\frac{\dot{H}}{H}+\frac{1}{2} \lambda \frac{H}{\mu+\Lambda} \dot{H} & =0 \Longrightarrow\left(\ln \frac{\mu+\Lambda}{H}\right)^{\cdot}+\frac{1}{2} \lambda \frac{H}{\mu+\Lambda} \dot{H}=0 \Longrightarrow \\
\left(\frac{\mu+\Lambda}{H}\right)^{\cdot}+\frac{1}{2} \lambda \dot{H} & =0 \Longrightarrow \\
\left(\mu+\frac{1}{2} \lambda H^{2}\right) & =0
\end{aligned}
$$

This equation means that the energy of the EM string fluid is constant along the flow lines of the observers. Working similarly we show that equation (119) becomes:

$$
\left(\mu+\frac{1}{2} \lambda H^{2}\right)^{*}=0
$$

and implies that the total energy of the magnetofluid is conserved along the magnetic field lines. Both these results are compatible with:

a. The fact that the magnetic field lines are frozen along the flow lines of the fluid (there is no relative motion of the two sets of lines) due to the condition $N^{a}=0$

b. The dynamic equation $q_{\psi}^{a}=0$ i.e. there is no heat flux wrt the observers $u^{a}$. 
There remains equation (120). Taking into account the fact that $\xi(t)$ (hence $p_{b}^{a} \xi_{, a}=0$ ) we find that this equation becomes:

$$
\left(\mu+\Lambda+\frac{1}{2} \lambda H^{2}\right) p_{a}^{b} n^{*}=0 .
$$

Because the total energy of the fluid (including the cosmological constant) is considered to be positive this equation gives the condition:

$$
p_{a}^{b} n^{*}=0 .
$$

This is a dynamical equation which involves the magnetic field only. However we also have $n^{*} u_{a}=$ $-\sigma_{a b} n^{a} n^{b}-\frac{\theta}{3}=0$. But since $\sigma_{a b}=0$ we find that ${ }^{*}{ }^{a} u_{a}=-\frac{\theta}{3}$ which from $p_{a}^{b} n^{*}=0$ gives

$$
n^{*}=\frac{1}{3} \theta u^{a}=(\ln R)^{\cdot} u^{a} .
$$

Eventually we have that the magnetic field lines are carried along with the fluid so that the total energy density (that is fluid energy and magnetic field energy) remains constant. Furthermore the fluid does not heat.

The magnetic field lines are coplanar with the fluid lines but they are not Lie transported along these lines except in the case of Minkowski spacetime. Indeed from the condition $N^{a}=0$ we have $L_{u} n^{a}=a u^{a}+b n^{a}$ where $a, b$ are quantities which have to be computed. From the definition of the Lie derivative we have $L_{u} n^{a}=\dot{n}^{a}-u^{*}$ therefore we have:

$$
\dot{n}^{a}-u^{*}=a u^{a}+b n^{a} .
$$

Contracting in turn with $u^{a}, n^{a}$ we find $a=(\ln \xi)^{*}, b=-\frac{1}{3} \theta$ therefore:

$$
\mathcal{L}_{u} n^{a}=(\ln \xi)^{*} u^{a}-\frac{1}{3} \theta n^{a}
$$

which proves our assertion. From this relation it follows that $p_{b}^{a} L_{u} n^{a}=0$, that is $N^{a}=0$.

Concerning the magnetic field we have

$$
L_{u} H^{a}=\dot{H}^{a}-\stackrel{u}{*}^{a} H=\dot{H} n^{a}+H \mathcal{L}_{u} n^{a}=(\ln \xi)_{, b} H^{b} u^{a}-\theta H^{a} .
$$

\section{The EMSF in spacetimes admitting a spacelike CKV $\xi^{a}=\xi n^{a}$}

We derive again the kinematic and the dynamic equations as we did for the case of $\xi^{a}=\xi u^{a}$.

\subsection{The Kinematic conditions of a spacelike CKV $\xi^{a}=\xi n^{a}$}

For a double congruence $u^{a}, n^{a}$ one has the kinematic quantities $\sigma_{a b}, \omega_{a b}, \theta, \dot{u}_{a}$ for the timelike congruence $u^{a}$ and the kinematic quantities $\mathcal{S}_{a b}, \mathcal{R}_{a b}, \mathcal{E} \dot{n}_{a}, \stackrel{*}{u}_{a}$ for the spacelike congruence $n^{a}$. Therefore the kinematic restrictions in this case involve in general all nine quantities plus the parameters $\psi$ and $H_{a b}$ and their derivatives. To find the kinematic conditions resulting from a collineation relative to a double congruence we need the $1+1+2$ decomposition of $H_{a b}$. To do that we consider the symmetry defining equation and contract it to get:

$$
\psi=\frac{\xi}{4}\left[\mathcal{E}+(\ln \xi)^{*}-\dot{n}^{c} u_{c}\right]
$$

For the case of a CKV $\xi^{a}=\xi n^{a}$ we find the following kinematic conditions [27]:

Proposition 3 A fluid spacetime $u^{a}$ with a spacelike congruence $n^{a}\left(u^{a} n_{a}=0\right)$ admits the spacelike CK 诂 $\xi^{a}=\xi n^{a}(\xi>0)$ iff:

$$
\begin{aligned}
\mathcal{S}_{a b} & =0 \\
\dot{n}_{a} u^{a} & =-\frac{1}{2} \mathcal{E} \\
n^{*} & =(\ln \xi)^{\cdot} u^{a}-p^{a b}(\ln \xi)_{, b} \\
N_{a} & =-2 \omega_{a b} n^{b} .
\end{aligned}
$$

\footnotetext{
${ }^{5} \xi$ is not necessarily equal to $H$ !
} 
The conformal factor $\psi$ satisfies:

$$
\psi=\frac{1}{2} \xi \mathcal{E}=\stackrel{*}{\xi} .
$$

Furthermore we can show the Lie derivatives [22]:

$$
\begin{aligned}
& L_{\xi} n^{a}=-\psi n^{a} \\
& L_{\xi} u^{a}=-\psi u^{a}-\xi N^{a} .
\end{aligned}
$$

We note that:

$$
\mathcal{E}=\left(\ln \xi^{2}\right)^{*}
$$

Also $n^{*}$ is the principal normal to the magnetic field lines. We note that in general these lines are not straight lines. The main results on the kinematics of a CKV $\xi^{a}=\xi n^{a}$ are given in the following Proposition (see Theorem 4.1. of [23]):

Proposition 4 Let $\xi^{a}=\xi n^{a}$ be a spatial conformal Killing vector orthogonal to $u^{a}$. Then $L_{\xi} n_{a}=\psi n_{a}$. Furthermore the following statements are equivalent:

1. $N^{a}=0$

2. $\omega^{a} \| \xi^{a}$ or $\omega^{a}=0$

3. $L_{\xi} u_{a}=\psi u_{a}$

4. $L_{\xi} \omega_{a b}=\psi \omega_{a b}$

5. $L_{\xi} \sigma_{a b}=\psi \sigma_{a b}$

6. $L_{\xi} \dot{u}_{a}=\psi,_{a}+\dot{\psi} u_{a}$

7. $L_{\xi} \theta=-\psi \theta+3 \dot{\psi}$

We have the obvious identity:

$$
\dot{n}^{a}=-\left(\dot{n}_{b} u^{b}\right) u^{a}+p_{b}^{a} \dot{n}^{b}
$$

We also have:

$$
\begin{aligned}
N^{a} & =p_{b}^{a}\left(\mathcal{L}_{u} n^{b}\right)=p_{b}^{a}\left(\dot{n}^{b}-\stackrel{u}{u}^{b}\right)=p_{b}^{a} \dot{n}^{b}-p_{b}^{a} u^{b} \\
& =p_{b}^{a} \dot{n}^{b}-p_{b}^{a}\left(\sigma_{c}^{b}+\omega_{c}^{b}\right) n^{c}=p_{b}^{a} \dot{n}^{b}-p_{b}^{a} \sigma_{c}^{b} n^{c}-\omega^{a}{ }_{c} n^{c} \\
& =p_{b}^{a} \dot{n}^{b}-p_{b}^{a} \sigma_{c}^{b} n^{c}+\frac{1}{2} N^{a} \Rightarrow \\
p_{b}^{a} \dot{n}^{b} & =p_{b}^{a} \sigma_{c}^{b} n^{c}+\frac{1}{2} N^{a} .
\end{aligned}
$$

Using the symmetry equation we find:

$$
\dot{n}^{a}=\frac{1}{2} \mathcal{E} u^{a}+p_{b}^{a} \sigma_{c}^{b} n^{c}+\frac{1}{2} N^{a} .
$$

\subsection{The dynamic conditions of a spacelike CKV $\xi^{a}=\xi n^{a}$}

We have to consider three sets of equations i.e. Maxwell equations, the conservation equations and the gravitational field equations. 


\subsubsection{Maxwell equations}

The above results hold for any spacelike CKV and any string fluid. For the particular case of the EMSF we have to supplement these equations with Maxwell equations which are

$$
\begin{aligned}
N^{a} & =0 \Longleftrightarrow p_{b}^{a} \dot{n}^{b}=p_{. c}^{a} \sigma_{b}^{c} n^{b} \\
\mathcal{E} & =-(\ln H)^{*} \\
\sigma_{a b} n^{a} n^{b}-\frac{2}{3} \theta & =(\ln H) \\
e & =2 \omega^{a} H_{a}, \quad \mathcal{R}^{a}=-\frac{j^{b} H_{b}}{2 H^{3}} H^{a} .
\end{aligned}
$$

Using $\mathcal{E}=-(\ln H)^{*}$ and (141) we find:

$$
\left(\xi^{2} H\right)^{*}=0
$$

that is the quantity $\xi^{2} H$ is constant along the magnetic field lines.

We also conclude that $\omega^{a} \| H^{a}$ that is the magnetic field congruence coincides with the vorticity congruence.

Using Maxwell equations we show the following important Proposition.

Proposition $5 \xi^{a}$ is a $C K V$ of the screen metric $p_{a b}$ as well as of the 3-metric $h_{a b}$ with conformal factor $\psi=\frac{1}{2} \xi \mathcal{E}$ (the same for both metrics).

Proof

In 29] it has been shown (see relations $(26),(27)$ ) that the following general relations/identities hold for the Lie derivatives of the projection tensors $h_{a b}$ and $p_{a b}$ :

$$
\begin{aligned}
& \frac{1}{\xi} L_{\xi} p_{a b}=2\left(\mathcal{S}_{a b}+\frac{1}{2} \mathcal{E} p_{a b}\right)-2 u_{(a} N_{b)} \\
& \frac{1}{\xi} L_{\xi} h_{a b}=2\left(\mathcal{S}_{a b}+\frac{1}{2} \mathcal{E} p_{a b}\right)-2 u_{(a} N_{b)}+2(\ln \xi)_{,(a} n_{b)}+2 n_{(a}^{*} n_{b)} .
\end{aligned}
$$

From equation (144) and the kinematic condition (134) these equations reduce as follows:

$$
\begin{aligned}
\frac{1}{\xi} L_{\xi} p_{a b} & =\mathcal{E} p_{a b} \\
\frac{1}{\xi} L_{\xi} h_{a b} & =\mathcal{E} p_{a b}+2(\ln \xi)_{,{ }_{a}} n_{b)}+2 n_{(a}^{*} n_{b)} .
\end{aligned}
$$

It follows immediately that $\xi^{a}$ is a CKV for the 2-metric $p_{a b}$ in the screen space with conformal factor $\frac{1}{2} \xi \mathcal{E}$.

To show that $\xi^{a}$ is a CKV for the 3 -metric $h_{a b}$ we $1+1+2$ decompose $(\ln \xi)_{, a}$ in terms of the vectors $u^{a}, n^{a}$ and find:

$$
(\ln \xi)_{, a}=-(\ln \xi)^{\cdot} u_{a}+(\ln \xi)^{*} n_{a}+p_{a}^{c}(\ln \xi)_{, c} .
$$

From (151) and (136) we have then:

$$
\begin{aligned}
\frac{1}{\xi} L_{\xi} h_{a b} & =\mathcal{E} p_{a b}+2(\ln \xi)_{,(a} n_{b)}+2{\stackrel{*}{n_{(a}} n_{b)}}^{*} \\
& =\mathcal{E} p_{a b}+2\left[{ }^{*}{ }_{d}-(\ln \xi)^{\cdot} u_{d}+(\ln \xi)^{*} n_{d}+p_{d}^{c}(\ln \xi)_{, c}\right] \delta_{(a}^{d} n_{b)} \\
& =\mathcal{E} p_{a b}+2(\ln \xi)^{*} n_{a} n_{b} .
\end{aligned}
$$

But from (138) we have that $\mathcal{E}=2(\ln \xi)^{*}$ therefore:

$$
\frac{1}{\xi} L_{\xi} h_{a b}=\mathcal{E}\left(p_{a b}+n_{a} n_{b}\right)=\mathcal{E} h_{a b}
$$

from which it follows that $\xi^{a}$ is a CKV for the 3 -metric $h_{a b}$ with conformal factor $\frac{1}{2} \xi \mathcal{E}$. 
From (143) we also have6:

$$
\dot{n}^{a}=-\frac{1}{2}(\ln H)^{*} u^{a}+p_{b}^{a} \sigma_{c}^{b} n^{c} .
$$

\subsubsection{Conservation equations}

These equations are the same as before, that is we have:

$$
\begin{aligned}
\dot{\mu}-\left(\mu+p_{\perp}\right)(\ln H)^{\cdot} & =0 \\
\stackrel{*}{\mu}-\left(\mu+p_{\perp}\right)(\ln H)^{*} & =0 \\
p_{a}^{b}\left[p_{\perp, b}+\lambda H H_{, b}+\left(\mu+p_{\perp}+\lambda H^{2}\right)\left(\dot{u}_{b}-\stackrel{*}{n}_{b}\right)\right] & =0 .
\end{aligned}
$$

\subsubsection{Gravitational field equations}

We use (98) to compute these equations. Of course we can also take them directly from [29] but we prefer to derive them here in order to make clear the methods we follow.

First we compute the $L_{\xi} R_{a b}$. We note that:

$$
p_{\psi}=\frac{1}{3} \psi_{; a b} h^{a b}=\frac{1}{3} \psi_{; a b}\left(p^{a b}+n^{a} n^{b}\right)=\frac{1}{3}\left(\gamma_{\psi}+a_{\psi}\right) .
$$

We have:

$$
\begin{aligned}
L_{\xi} R_{a b}= & -2 \psi_{; a b}-g_{a b} \square \psi \\
= & -2\left[\lambda_{\psi} u_{a} u_{b}+2 k_{\psi} u_{(a} n_{b)}+2 \mathcal{S}_{\psi(a} u_{b)}+\gamma_{\psi} n_{a} n_{b}+2 P_{\psi(a} n_{b)}+\frac{1}{2} \alpha_{\psi} p_{a b}+D_{\psi a b}\right]- \\
& -\left(-\lambda_{\psi}+3 p_{\psi}\right)\left(-u_{a} u_{b}+n_{a} n_{b}+p_{a b}\right) \\
= & 3\left(p_{\psi}-\lambda_{\psi}\right) u_{a} u_{b}+\left(\lambda_{\psi}-3 p_{\psi}-2 \gamma_{\psi}\right) n_{a} n_{b}+\left(\lambda_{\psi}-3 p_{\psi}-a_{\psi}\right) p_{a b}+\text { rest. }
\end{aligned}
$$

From (98) we get the following field equations (including equations $k_{\psi}=0, S_{\psi a}=0, P_{\psi a}=0, D_{\psi a b}=0$ which result directly from the kinematic conditions over (98)):

$$
\begin{aligned}
p_{\perp}^{*}+\lambda H \stackrel{*}{H}+2\left(p_{\perp}+\frac{1}{2} \lambda H^{2}-\Lambda\right) \frac{1}{2} \mathcal{E} & =\frac{1}{\xi} 3\left(p_{\psi}-\lambda_{\psi}\right) \\
p_{\perp}^{*}+\lambda H \stackrel{*}{H}+2\left(p_{\perp}+\frac{1}{2} \lambda H^{2}-\Lambda\right) \frac{1}{2} \mathcal{E} & =-\frac{1}{\xi}\left(\lambda_{\psi}-3 p_{\psi}-2 \gamma_{\psi}\right) \\
\stackrel{*}{\mu}+\lambda H \stackrel{*}{H}+\left(\mu+\frac{1}{2} \lambda H^{2}+\Lambda\right) \mathcal{E} & =\frac{1}{\xi}\left(\lambda_{\psi}-3 p_{\psi}-a_{\psi}\right) .
\end{aligned}
$$

Using equation (145) to replace $\mathcal{E}$ in terms of $(\ln H)^{*}$ we note that the first two equations have identical lhs and they result in the two equations:

$$
\begin{aligned}
p_{\perp}^{*}-\left(p_{\perp}-\frac{1}{2} \lambda H^{2}-\Lambda\right)(\ln H)^{*} & =\frac{1}{\xi} 3\left(p_{\psi}-\lambda_{\psi}\right) \\
\lambda_{\psi} & =-\gamma_{\psi} .
\end{aligned}
$$

The last equation is written:

$$
\stackrel{*}{\mu}-\left(\mu-\frac{1}{2} \lambda H^{2}+\Lambda\right)(\ln H)^{*}=\frac{1}{\xi}\left(\lambda_{\psi}-3 p_{\psi}-a_{\psi}\right) .
$$

\footnotetext{
${ }^{6}$ One could possibly expect to get information on $\sigma_{b c} n^{b} n^{c}$ from this equation. But this is not so. Indeed by expanding $p_{b}^{a}$ we find:

and we get no information on $\sigma_{b c} n^{b} n^{c}$.

$$
\dot{n}^{a}=-\frac{1}{2}(\ln H)^{*} u^{a}+\sigma_{c}^{a} n^{c}-\left(\sigma_{b c} n^{b} n^{c}\right) n^{a}
$$
}


Using the conservation equation (153) and equation (155) we find:

$$
\left(p_{\perp}+\frac{1}{2} \lambda H^{2}-\Lambda\right)(\ln H)^{*}=-\frac{2}{\xi}\left(\gamma_{\psi}+a_{\psi}\right)
$$

Finally we have that the field equations in the case of a spacelike vector $\xi^{a}=\xi n^{a}$ are:

$$
\begin{aligned}
p_{\perp}^{*}+\lambda H \stackrel{*}{H} & =\frac{1}{\xi}\left(2 \gamma_{\psi}-a_{\psi}\right) \\
\left(p_{\perp}+\frac{1}{2} \lambda H^{2}-\Lambda\right)(\ln H)^{*} & =-\frac{2}{\xi}\left(\gamma_{\psi}+a_{\psi}\right) .
\end{aligned}
$$

where:

$$
\psi_{; a b}=-\gamma_{\psi}\left(u_{a} u_{b}-n_{a} n_{b}\right)+\frac{1}{2} \alpha_{\psi} p_{a b} .
$$

We see that $\psi_{; a b}$ is the energy momentum tensor or equivalently the Ricci tensor of a string fluid with $\rho=-\gamma_{\psi}$ and $q=\frac{1}{2} \alpha_{\psi}$ or vice versa. Obviously one can make many scenarios with this observation.

The result we found coincides with the one we found in [29] on strings.

From equations (157) and (158) ones shows easily that:

$$
\left[\left(p_{\perp}+\frac{1}{2} \lambda H^{2}-\Lambda\right) H\right]^{*}=-\frac{3 H}{\xi} \alpha_{\psi}
$$

This equation shows that if $\alpha_{\psi}=p^{a b} \psi_{; a b}=0$ then the quantity $\left(p_{\perp}+\frac{1}{2} \lambda H^{2}-\Lambda\right) H$ is constant along the magnetic field lines.

The constraint equation 7 for a general anisotropic fluid of the form we consider is:

$$
\left(\mu-2 p_{\perp}+p_{\|}+2 \Lambda\right) \psi=2\left(2 \lambda_{\psi}-a_{\psi}\right) .
$$

Setting $\mu=-p_{\| 1}$ and $p_{\perp}=p_{\perp}+\frac{1}{2} \lambda H^{2}$ we obtain the EMSF. In this case equation (161) becomes:

$$
\left(p_{\perp}+\frac{1}{2} \lambda H^{2}-\Lambda\right) \psi=a_{\psi}+2 \gamma_{\psi}
$$

But $\psi=\frac{1}{2} \xi \mathcal{E}=-\frac{1}{2} \xi(\ln H)^{*}$ therefore we obtain the same result.

We collect the above results in the following Proposition.

Proposition 6 An EMSF spacetime admits a CKV of the form $\xi^{a}=\xi n^{a}$ where $n^{a}=H^{a} / H$ iff the following

\footnotetext{
${ }^{7}$ This equation follows form the identity $\left(R^{a b} \xi_{b}\right)_{; a}=-3 \square \psi$ which holds for all CKVs.
} 
system of equations is satisfied:

$$
\begin{aligned}
\dot{\mu}-\left(\mu+p_{\perp}\right)(\ln H)^{\cdot} & =0 \\
\dot{*}-\left(\mu+p_{\perp}\right)(\ln H)^{*} & =0 \\
p_{a}^{b}\left[\left(p_{\perp}+\frac{1}{2} \lambda H^{2}-\Lambda\right)_{, b}+\left(\mu+p_{\perp}+\lambda H^{2}\right)\left(\dot{u}_{b}-\stackrel{*}{n}_{b}\right)\right]^{\prime} & =0 \\
\psi_{; a b} & =-\gamma_{\psi}\left(u_{a} u_{b}-n_{a} n_{b}\right)+\frac{1}{2} \alpha_{\psi} p_{a b} \\
{\left[\left(p_{\perp}+\frac{1}{2} \lambda H^{2}-\Lambda\right)\right]^{*} } & =-\frac{3 H}{\xi} \alpha_{\psi} \\
\left(p_{\perp}+\frac{1}{2} \lambda H^{2}-\Lambda\right)(\ln H)^{*} & =-\frac{2}{\xi}\left(\gamma_{\psi}+a_{\psi}\right) \\
\mathcal{S}_{a b} & =0, \mathcal{E}=-(\ln H)^{*} \\
\dot{n}^{a} & =-\frac{1}{2}(\ln H)^{*} u^{a}+p_{b}^{a} \sigma_{c}^{b} n^{c} \\
\dot{*}^{a} & =(\ln \xi)^{*} n^{a}-(\ln \xi)^{, a} \\
N_{a} & =0 \\
\sigma_{a b} n^{a} n^{b}-\frac{2}{3} \theta & =(\ln H)^{.} \\
e & =2 \omega^{a} H_{a}, \quad \mathcal{R}^{a}=-\frac{j^{b} H_{b}}{2 H^{3}} H^{a} .
\end{aligned}
$$

Furthermore the rotation $\omega^{a}$ is either parallel to $H^{a}$ or vanishes and $\psi=\frac{1}{2} \xi \mathcal{E}=-\frac{1}{2} \xi(\ln H)^{*}$.

One important result is that if the vorticity vanishes then the same must be true for the charge density and conversely. This is a restriction of physical nature resulting from a geometrical symmetry assumption.

A CKV for which $\psi_{; a b}=0$ is called a special CKV. Coley and Tupper [26] have shown that if an anisotropic fluid space-time admits a proper special $\mathrm{CKV} \xi^{a}=\xi n^{a}$ then (assuming $\Lambda=0$ ):

$$
\mu=-p_{\|}=\frac{1}{2} R, p_{\perp}=0
$$

where $R$ is the Ricci scalar. From Einstein field equations it follows that for this case $T_{a b}$ is of the form:

$$
T_{a b}=\frac{1}{2} R\left(u_{a} u_{b}-n_{a} n_{b}\right) .
$$

For the case of a string fluid this result gives $\rho=\frac{1}{2} R$ and $q=0$. Obviously $R \neq 0$ otherwise we do not have a fluid at all. Let us check if our results are compatible with this general result.

From equation (168) assuming $\stackrel{*}{H} \neq 0$ we have:

$$
p_{\perp}+\frac{1}{2} \lambda H^{2}-\Lambda=0
$$

which gives immediately from (45):

$$
R_{a b}=\left(\mu+\frac{1}{2} \lambda H^{2}+\Lambda\right) p_{a b}
$$

from which follows:

$$
R=2\left(\mu+\frac{1}{2} \lambda H^{2}+\Lambda\right)
$$

Therefore:

$$
R_{a b}=\frac{R}{2} p_{a b} .
$$


Consequently due to the symmetry between the Ricci tensor and the energy momentum tensor for a string fluid:

$$
T_{a b}=\frac{R}{2}\left(u_{a} u_{b}-n_{a} n_{b}\right)
$$

which is in agreement with the quoted result.

Proposition 7 Let $\xi^{a}=\xi n^{a}$ be a proper special CKV in an EMSF space-time and let the total energy of the magnetofluid $\mu+\frac{1}{2} \lambda H^{2}+\Lambda \neq 0$. Then for $\stackrel{*}{H} \neq 0$ we have the following

(a) The Ricci tensor satisfies the property 8 :

$$
R_{a b}=\frac{R}{2} p_{a b}
$$

(b) The quantity $R / H$ is constant along the magnetic field lines and along the fluid lines and

(c) The following equations hold:

$$
\begin{aligned}
\stackrel{*}{\mu}-\left(\mu-\frac{1}{2} \lambda H^{2}+\Lambda\right)(\ln H)^{*} & =0 \\
\dot{\mu}-\left(\mu+p_{\perp}\right)(\ln H)^{*} & =0
\end{aligned}
$$

together with equations (169) - 174).

Proof

The first part (a) has been shown above.

Concerning (b) we note that (164) can be written:

$$
\begin{aligned}
\stackrel{*}{\mu}-\left(\mu-\frac{1}{2} \lambda H^{2}+\Lambda\right)(\ln H)^{*} & =0 \Longrightarrow \\
\stackrel{*}{\mu}-\left(\frac{R}{2}-\lambda H^{2}\right)(\ln H)^{*} & =0 \Longrightarrow \\
\stackrel{*}{\mu}+\lambda H H^{*}-\frac{R}{2}(\ln H)^{*} & =0 \Longrightarrow \\
\left(\mu+\frac{1}{2} \lambda H^{2}+\Lambda\right)^{*}-\frac{R}{2}(\ln H)^{*} & =0 \Longrightarrow \\
(\ln R)^{*}-(\ln H)^{*} & =0 \Longrightarrow \\
\left(\ln \frac{R}{H}\right)^{*} & =0
\end{aligned}
$$

from which follows that the quantity $R / H$ is constant along the magnetic field lines.

Working in exactly the same way we show that $(\ln (R / H))^{\circ}=0$ from which follows that the quantity $R / H$ is constant along the fluid flow line.

Concerning the case of KVs we have the following result.

Proposition 8 An EMSF spacetime admits a $K V$ of the form $\xi^{a}=\xi n^{a}$ where $n^{a}=H^{a} / H$ iff

(a) $\stackrel{*}{H}=\stackrel{*}{\xi}=0$

(b) The following equations hold

$$
\begin{aligned}
\stackrel{*}{\mu}^{*} & =p_{\perp}^{*}=0 \\
\dot{\mu}-\left(\mu+p_{\perp}\right)(\ln H)^{\cdot} & =0 \\
\mathcal{S}_{a b} & =0, \mathcal{R}^{a}=-\frac{j^{b} H_{b}}{2 H^{3}} H^{a} \\
\stackrel{*}{n}^{a} & =-(\ln \xi)^{a} \\
\dot{n}^{a} & =p_{b}^{a} \sigma_{c}^{b} n^{c} \\
\sigma_{a b} n^{a} n^{b}-\frac{2}{3} \theta & =(\ln H)^{.} \\
N_{a} & =0, \quad e=2 \omega^{a} H_{a} .
\end{aligned}
$$

\footnotetext{
${ }^{8}$ It is not necessarily an Einstein space!
} 
We conclude that when an EMSF admits the $\mathrm{KV} \xi^{a}=\xi n^{a}$ the following results hold:

i) Because $n^{a}=\omega^{a} / \omega=H^{a} / H(\omega \neq 0)$ the string consists of the 2-dimensional timelike surface spanned by $u^{a}$ and the vorticity $\omega^{a}$ (Nambu geometric string) or $\omega^{a}=0$.

ii) From equations (148), (149) it follows:

$$
L_{\xi} p_{a b}=0, \quad L_{\xi} h_{a b}=0
$$

that is, $\xi^{a}$ is also a KV of the metric $h_{a b}$ of the 3 -space normal to $u^{a}$, and a KV of the screen space metric $p_{a b}$.

iii) From Proposition 4 we have that the Killing symmetry is inherited by the geometric and the dynamic variables, that is:

$$
L_{\xi} u_{a}=L_{\xi} n_{a}=0, \quad L_{\xi} \sigma_{a b}=0, L_{\xi} \omega_{a b}=0, L_{\xi} \theta=0, L_{\xi} \dot{u}_{a}=0 .
$$

iv) If $\omega^{a} \neq 0$ then $u^{a}, n^{a}=\omega^{a} / \omega$ must commute.

Obviously these restrictions are severe and allow only few special choices for the string fluids in given spacetimes.

\subsection{Application: EMSF in Bianchi I spacetime}

The Bianchi I spacetime with metric

$$
d s^{2}=-d t^{2}+A_{1}^{2}(t) d x^{2}+A_{2}^{2}(t) d y^{2}+A_{3}^{2}(t) d z^{2} .
$$

has been a platform for studying anisotropy and more specifically string fluids and electromagnetic fields. For example Letelier [9] studied string dust in Bianchi I spacetime whereas the electromagnetic field in the relativistic RMHD has been studied (among many others) in [28. Following this line of research we shall use the results obtained in the last section to compute all possible Bianchi I spacetimes (if any), which carry a magnetic field satisfying the RMHD approximation and admit a spacelike CKV or a spacelike KV.

In order to get comparable results with the literature we consider the comoving observers $u^{a}=(1,0,0,0)$. This choice has a double effect. On the one hand gives that the vorticity $\omega^{a}=0$, therefore Maxwell equation $e=2 \omega^{a} H_{a}$ implies that the charge density $e=0$. This excludes all analytical solutions found in [28. Secondly the geometric condition $N^{a}=0$ restricts heavily the possible symmetry vectors $\xi^{a}=\xi n^{a}$. All the CKVs of the Bianchi I spacetime have been found in 33.

We have checked that for this choice of $u^{a}$ none of these vectors satisfies the condition $N^{a}=0$. Therefore the only remaining choice is the KVs so that the system of equations we have to solve are equations (181) to $(? ?)$.

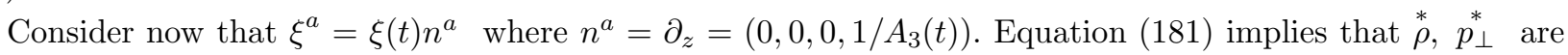
zero hence $\rho(t), p_{\perp}(t)$. We prove easily that equation (184) gives $\xi=1$ therefore the KV is the $\partial_{z}$. Equation (185) is satisfied identically, while equation (186) gives $H(t)=\left(A_{1}(t) A_{2}(t)\right)^{-1}$. Therefore the magnetic field is given by

$$
H^{a}=\left(A_{1}(t) A_{2}(t)\right)^{-1} \partial_{z}
$$

It remains equation (182) which is written as

$$
\dot{\rho}+\left(\rho+p_{\perp}\right) \ln \left[A_{1}(t) A_{2}(t)\right]^{\cdot}=0
$$

For each equation of state we determine a Bianchi I space time which admits a string fluid. For example, let us consider the eqn of state $p_{\perp}=\rho \neq 0$. Then from (40) we have that the EMSF has the enrgy momentum tensor

$$
\begin{aligned}
T_{a b} & =\left(\rho+\frac{1}{2} \lambda H^{2}\right) u_{a} u_{b}-\left(\rho+\frac{1}{2} \lambda H^{2}\right) n_{a} n_{b}+\underbrace{\left(\rho+\frac{1}{2} \lambda H^{2}\right)}_{\equiv q} p_{a b} \\
& =\left(\rho+\frac{1}{2} \lambda H^{2}\right)\left(u_{a} u_{b}-n_{a} n_{b}+p_{a b}\right)
\end{aligned}
$$

Eqn (191) gives

$$
\rho=\frac{c}{A_{1}(t) A_{2}(t)}
$$

therefore in the Bianchi I spacetime we know the string fluid as well as the magnetic field. 


\section{Conclusions}

We have applied the $1+1+2$ decomposition to the case of the EMSF in the RMHD approximation. We have shown that a geometric assumption in the form of a symmetry effects both the kinematics and the dynamics of the resulting EMSF. We have approached the problem in two steps a. In full generality independently of a particular symmetry and b. In the case of a CKV which is of the form $\xi^{a}=\xi u^{a}$ and of the form $\xi^{a}=\xi n^{a}$ with $n^{a}=H^{a} / H$ where $u^{a}$ is the four velocity of the fluid and $H^{a}$ is the magnetic field. We applied the results of the $\xi^{a}=\xi u^{a}$ case in the FRW spacetime and the results of the case $\xi^{a}=\xi n^{a}$ in the Bianchi I spacetime where. In the latter case we found new solutions for the gravitational field.

It is apparent that the results stated in this work due to their generality can be used in many different situations involving the electromagnetic field and various types of symmetries. However one may ask if all the initial conditions are viable for the solutions which follow from the existence of symmetries. In particular, the existence of a symmetry in a solution is a strong argument which its violations leads to another kind of solution. On the other hand, from the theory of similarity solutions of differential equations [35, 36] we know that for a given differential equation a similarity solution satisfy the initial value problem/boundary conditions iff the later are also invariant under the action of the symmetries which provide the similarity transformations. That property can be applied in order to define initial conditions where a nonsymmetric solution can be related with a symmetric one. For instance, to relate the inner and outer solutions in a compact body.

\section{Acknowledgement}

The authors thank the anonymous referees for their comments and suggestions which helped to improve the quality and the presentation of this work. AP acknowledges the financial support of FONDECYT grant no. 3160121 and thanks the University of Athens for the hospitality provided.

\section{References}

[1] E. Gourgoulhon, (2006) "An introduction to relativistic hydrodynamics" EAS Publ. Ser. 21, 43

[2] S. Grozdanov, D. M. Hofman and N. Iqbal (2017), "Generalized global symmetries and dissipative magnetohydrodynamics "Phys. Rev. D 95, 096003

[3] J. Hernandez and P. Kovtum (2017), "Relativistic Magnetohydrodynamics "JHEP 1705 (2017) 001

[4] J. Armas and A. Jain (2018), "Magnetohydrodynamics as superfluidity "arXiv:1808.01939 [hep-th]

[5] Letelier P (1979), Clouds of Strings in general Relativity, Phys. Rev. D 20, 1294

[6] L.L. Smally and J.P. Krisch (1996), String fluid dynamics, Class. Quantum Grav. 13, L19

[7] Ray D (1978) "Some solutions for relativistic vortices interacting through a scalar field" Phys Rev D, 18, $3879-3880$

[8] Lund F and Regge T (1976), "Unified approach to strings and vortices with soliton solutions" Phys Rev $\mathrm{D}, \mathbf{1 4}, 1524-1535$

[9] Letelier P (1980), "Anisotropic fluids with two perfect fluid components" Phys Rev D 22, 807 - 813

[10] Letelier P (1981), "” Nuovo Cimento B63, 519

[11] Letelier P (1983), "String Cosmologies" Phys Rev D 28, 2414 - 2419

[12] S. Grozdanov, D. M. Hofman and N. Iqbal (2017), "Generalized global symmetries and dissipative magnetohydrodynamics "Phys. Rev. D 95, 096003

[13] J. Hernandez and P. Kovtum (2017), "Relativistic Magnetohydrodynamics "JHEP 1705 (2017) 001

[14] J. Armas and A. Jain (2018), "Magnetohydrodynamics as superfluidity "[arXiv:1808.01939 [hep-th] 
[15] I Yavuz and I Yilmaz "Inheriting Conformal and Special Conformal Killing Vectors in String Cosmology" (1997) Gen Rel Grav 9, 1295 - 1307

[16] Yilmaz I (2001), "Timelike and Spacelike Ricci Collineation Vectors in String Cosmology" Inter Jour Modern Physics 10, 681 - 690

[17] Baysal H Yilmaz I (2002), "Spacelike Ricci Inheritance vectors in a model of string cloud and string fluid stress tensor" Class Quantum Grav 19, 6435 - 6443

[18] Baysal H, Camci U, Tarhan I and Yilmaz I (2002), "Conformal Collineations in String Cosmology" Inter Jour Modern Physics 11, 463 - 469

[19] Camci U (2002), "Conformal Collineations and Ricci Inheritance symmetry in String Cloud and String Fluids" Inter Jour Modern Physics 11, 353 - 366

[20] Sharif M Sheikh U (2005), "Timelike and Spacelike Matter Inheritance Vectors in Specific Forms of EnergyMomentum Tensor" Inter Jour Modern Physics (to appear), gr-qc/0504101

[21] Mason D P and Tsamparlis M 1985, "Spacelike Conformal Killing Vectors and Spacelike Congruences" J. Math. Phys. 26, 2881 - 2901

[22] Maartens R, Mason D P and Tsamparlis M 1986, "Kinematic and Dynamic Properties of Conformal Killing Vectors in Anisotropic Fluids" J. Math. Phys. 27, 2987 - 2994

[23] Saridakis E and Tsamparlis M 1991, "Symmetry Inheritance of Conformal Killing Vectors" J. Math. Phys. 32, $1541-1551$

[24] Noris L K, Green P. and Davis W R (1977), J. Math. Phys. 18, 1305

[25] Tsamparlis M 1992, "Geometrization of a General Collineation" J. Math. Phys. 33, 1472 - 1479

[26] Coley A.A and Tupper B. O. (1989) J. Math. Phys. 30, 2616

[27] Tsamparlis M and Mason D P 1990, "Ricci Collineation Vectors in Fluid Space-times" J. Math. Phys. 31, $1707-1722$

[28] Jacobs K (1969), "Cosmologies of Bianchi Type I with a Uniform Magnetic Field" Astro. Journal 155, 379 $-391$

[29] Tsamparlis M (2006), "General symmetries of a string fluid spacetime" Gen Rel Grav (2006)

[30] K.A. Dunn and B.O.J Tupper, Astroph. J. 235, 307 (1980)

[31] V.N. Duarte and R.A. Clement, J. Phys. Conf. Ser. 511, 012015 (2014)

[32] G.F.R. Ellis and H. van Elst and, Cosmological models, Cargèse Lectures (1998)

[33] M. Tsamparlis, A. Paliathanasis and L. Karpathopoulos, Gen. Relativ. Grav. 47, 15 (2015)

[34] M. Tsamparlis, "Special Relativity: An Introduction with 200 Problems and Solutions ", Springer, 2010

[35] P.E. Hydo, Symmetry analysis of initial-value problems, J. Math. Anal. Appl. 309, 103 (2005)

[36] R. Cherniha and S. Kovalenko, Lie symmetries of nonlinear boundary value problems, CNSNS 17, 71 (2012) 